\title{
LA CREATIVITÀ ARTISTICA QUALE STRUMENTO DI EMANCIPAZIONE CULTURALE. LETTERATURA E PITTURA DEGLI ITALIANI DI CROAZIA E SLOVENIA: L'ESEMPIO DI CLAUDIO UGUSSI
}

\author{
Elis Deghenghi Olujić \\ Università Juraj Dobrila di Pola \\ Dipartimento di studi in lingua italiana
}

\section{Riassunto}

L'articolo tratta della creatività letteraria e artistica degli appartenenti alla Comunità Nazionale Italiana $(\mathrm{CNI})$ di Croazia e Slovenia, che si manifesta nell'ambito del Concorso d'Arte e di Cultura «Istria Nobilissima» fondato nel 1967 dall'Università Popolare di Trieste e dall'Unione degli Italiani dell'Istria e di Fiume (dal 1991 Unione Italiana). Le Antologie delle opere premiate al Concorso, che si pubblicano annualmente dal 1968, costituiscono una fonte da consultare per conoscere le tappe del percorso di emancipazione culturale che gli italiani di Croazia e Slovenia hanno intrapreso e seguito dalla fine degli anni Sessanta dello scorso secolo a oggi. Inoltre, nell'anno in cui la Casa editrice EDIT di Fiume, fondata nel 1952, festeggia sessant'anni di attività, l'intervento pone in rilievo l'importante ruolo che l'Ente giornalistico-editoriale svolge a beneficio della Comunità. Si evidenzia in particolare il significato della pubblicazione, della ristampa e della diffusione delle opere degli autori istro-quarnerini, specialmente per il tramite della collana «Altre lettere italiane» avviata nel 2005 nella quale è evidente la volontà di coniugare la produzione letteraria e artistica degli italiani di Croazia e Slovenia per mezzo della scelta oculata della veste grafica dei volumi, curata da Daria Vlahov Horvat. Il contributo presenta inoltre un'analisi critica del romanzo La città divisa di Claudio Ugussi, che si presenta nella duplice veste di narratore e di pittore.

Parole chiave: letteratura, pittura, cultura, creatività, romanzo, storia individuale e collettiva

\section{Cenni introduttivi}

Si ritiene opportuno iniziare il discorso riassumendo la definizione della cultura data dall'UNESCO, specialmente per le sue implicazioni di ordine sociologico. La cultura, per l'Organizzazione delle Nazioni Unite, rappresenta una serie di caratteristiche specifiche di una società o di un gruppo sociale in termini spirituali, materiali, intellettuali ed emozionali. Se partiamo dalla premessa che la cultura va intesa nell'accezione 
antropologica di senso "totale", che comprende l'insieme delle espressioni artistiche, degli usi, dei costumi, delle credenze, dei simboli e dei valori che nel corso dei secoli gli uomini hanno prodotto, coltivato e preservato nel loro spazio vitale, si può affermare che ogni territorio ha una "sua" cultura. Anche l'Istria, la più estesa penisola della Croazia, spazio vitale della comunità di cui tratta quest'articolo, ha una "sua" cultura, composita e articolata. Difatti, questo lembo di terra non troppo grande, di una singolare forma triangolare, è stato nei secoli ed è tuttora punto d'incontro di genti, di tradizioni, di lingue e dialetti diversi. In questa particolare condizione storica, sociale e culturale, caratterizzata dalla presenza nello stesso territorio di popolazioni diverse, detentrici di differenti retaggi culturali, si è sviluppato un patrimonio culturale che va salvaguardato, perché rappresenta la testimonianza più tangibile della natura specifica, multiculturale e multietnica, della penisola istriana, che nei secoli è stata un palinsesto su cui tante mani hanno lasciato una traccia più o meno profonda. Senza il timore di usare termini eccessivamente laudativi, questo patrimonio culturale può definirsi unico, perché tale appare quando lo si considera con la dovuta attenzione anche nell'ambito del pur variegato e ricco panorama culturale europeo. Piccolo specchio dell'universo, il microcosmo istriano, nel quale il meticciamento di lingue e di culture esiste da secoli, è magico frammento della molteplicità europea, un vero e proprio laboratorio delle diversità. Un paradigma di un'Europa delle culture che nell'esperienza della realtà istriana potrebbe trovare quasi un modello cui ispirarsi per il proprio futuro.

In questo spazio geograficamente piccolo ma ricco dal punto di vista antropologico, l'identità del singolo e della collettività è alimentata da uno scambio culturale reciproco, persistente e amoroso, che arricchisce chi lo sperimenta nella prassi quotidiana. Gli abitanti di questa penisola d'inusitata ricchezza culturale, mistilingue e plurietnica, sono pacificamente consapevoli della propria diversità, si riconoscono nell'orgoglio della comune appartenenza al territorio e nel sentimento dell'istrianità. Il patrimonio culturale locale rappresenta una risorsa comune e un fattore di sviluppo importante per tutti gli abitanti della regione, un elemento imprescindibile della coscienza collettiva. Esso è un valido strumento per l'affermazione dell'identità regionale istriana, ed è un terreno fertile per gli studiosi della regione impegnati oggi, come in passato, nei più svariati campi di ricerca: etnografico, storico, antropologico, sociologico, dialettologico, pragmalinguistico, letterario.

Nelle Repubbliche di Croazia e Slovenia vive un'esigua comunità di italiani. Gli italiani, che in Croazia vivono principalmente in Istria e a Fiume, inseriti a fianco della popolazione di maggioranza croata in un ambiente plurietnico e multiculturale per storia e tradizioni, sono una presenza autoctona. Sono rimasti a vivere nella penisola istriana e nella città quarnerina al momento dell'esodo massiccio della popolazione di lingua e cultura italiana durato, con momenti di maggiore o minore intensità, dal 1943 al 1956, e protrattosi fino ai primi anni Sessanta dello scorso secolo. L'esodo è stato un fenomeno imponente e devastante, che nel secondo dopoguerra ha cambiato radicalmente il tessuto demografico della penisola istriana, e la cui conseguenza è stata la quasi totale cancellazione della presenza italiana in una terra in cui essa era presenza secolare. Proprio perché si è svolto in tempi rapidi, causando profonde lacerazioni che 
hanno sconvolto gli equilibri etnici e sociali, l'esodo ha determinato un volto nuovo dell'Istria, di Fiume e della Dalmazia. Rimasti a vivere nello spazio del loro insediamento storico come presenza autoctona dopo la firma del Trattato di Parigi e la sua ratifica nel gennaio del 1947, gli italiani "rimasti", divenuti minoranza, sono riusciti a conservare la propria identità nazionale e culturale soprattutto attraverso una intensa e poliedrica attività artistica, che spazia dalla letteratura al teatro e alla musica, dalla pittura alla scultura, dalla fotografia al design. L'apporto dato dai poeti, dai narratori, dai musicisti, dagli scultori, dai pittori, dai fotografi, dagli intellettuali italiani tout court al patrimonio culturale istro-quarnerino è considerevole. La loro produzione artistica corrobora questo patrimonio e favorisce al contempo un dialogo e un confronto qualificato con la maggioranza. Un dialogo che con l'apporto critico di analisi e visioni si propone come stimolo all'emanciparsi e all'evolversi di un'identità sociale, culturale e umana.

Pur avendo la sua genesi in un territorio ben definito, anche dal punto di vista geografico, l'attività artistica degli italiani di Croazia e Slovenia è tutt'altro che provinciale o marginale. Essa nasce e si alimenta dalla persuasione che l'universalità non è qualcosa di astratto, di esteriormente esemplificativo, ma scaturisce invece dall'approfondimento di una particolare condizione spazio temporale e dalla sua proiezione in una sfera più vasta, nella quale ognuno possa riconoscersi. Ma per quanto abbiano un'evidente valenza universale, le testimonianze artistiche degli italiani di Croazia e Slovenia riconducono sempre all'Istria, cifra della loro vita, terra magica vissuta come crocevia di culture, luogo di confronto e di integrazione di genti che le politiche e le guerre hanno diviso, ma che proprio la cultura ha unito e può continuare a unire in quello che è oggi un fenomeno europeo di tipo non escludente, ma unificante. Ed è la cultura, intesa come «tenuta etnica» ${ }^{1}$, il segno vincente degli italiani di Croazia e Slovenia. Perché una minoranza che non produca cultura è condannata all'estinzione, è destinata a trasformarsi in una presenza meramente folkloristica, nell'accezione meno nobile del termine. La creazione, invece, «di nuovi prodotti dell'intelletto e della fantasia dei singoli appartenenti alla esigua comunità italiana in Istria e a Fiume rilanciano quest'ultima come comunità "culturale", come comunità, cioè, che produce cultura, ridefinendo i confini oggi esistenti tra "folklore" (nel suo significato di "recupero") e "cultura" (nel significato di "creatività") $\rangle^{2}$. Nello specifico, la creatività artistica degli appartenenti alla Comunità Nazionale Italiana va intesa come testimonianza della sua vitalità. Attraverso i suoi artisti e intellettuali questa comunità difatti «recupera i propri valori guardando avanti e cercando di affermarsi nello spazio del suo insediamento storico, ma anche al di fuori di esso, con particolare riferimento ai paesi di residenza (Croazia e Slovenia) e all'Italia» ${ }^{3}$. È questo il modo migliore per la Comunità Nazionale Italiana di essere considerata e percepita dalla maggioranza croata e slovena ma anche dagli italiani d'Italia non come una mera presenza folkloristica, sopravvissuta agli eventi a conclusione della Seconda guerra mondiale e rimasta quale timida espressione residua,

1 Damiani, A., "La cultura come «tenuta etnica»", in «La Battana», trimestrale di cultura, anno XL, n. 147, EDIT, Fiume, 2003, p. 33.

2 Monica, L., "La comunità italiana in Istria tra folklore e cultura", in Civiltà italiana e geografie d'Europa (a cura di Bianca Maria Da Rif), EUT Edizioni Università di Trieste, Trieste, 2009, p. 158.

3 Ibidem. 
ma come una comunità vitale, che per il tramite della sua attività nel campo artistico assicura «la prosecuzione della presenza etnica e culturale nel territorio in posizione non subalterna agli altri coinquilini» ${ }^{4}$. L'originale produzione artistica degli italiani di Croazia e Slovenia non è più finalizzata, come in passato, a difendere esclusivamente l'identità. Pertanto essa si configura oggi come un atto di fede nella creatività umana e come documento di quella vita che «si svolge, con proprie connotazioni politiche, ideologiche, psicologiche, morali, ambientali, nel territorio istriano» ${ }^{5}$. Un territorio nel quale la Comunità Nazionale Italiana, come tutte le altre minoranze che vi risiedono, rappresenta una ricchezza da tutelare, un'opportunità che i Paesi di residenza e quello d'origine devono cogliere per far convivere etnie, lingue e culture in armonia con la visione europea, che garantisce a tutte le minoranze il diritto di esprimersi secondo il principio delle "pari opportunità".

\section{Il Concorso d'Arte e di Cultura "Istria Nobilissima"}

Gli anni Sessanta dello scorso secolo, nonostante le non facili condizioni politiche e sociali, per gli italiani di Croazia e Slovenia sono stati generosi di promettenti iniziative che hanno impresso una salutare spinta sinergica alle loro espressioni artistiche. Vanno ricordati pertanto come un momento felice, specialmente «in virtù del fatto che la minoranza riuscì a darsi una serie di organismi volti alla produzione di cultura e al mantenimento dell'identità» ${ }^{6}$. In quegli anni difatti «si consolidarono, nacquero o vennero gettate le basi di nuove strutture mass-mediologiche, scientifiche e letterario-artistiche sulle quali tutt'oggi poggia, assieme alla rete scolastica, la vita culturale della minoranza italiana» ${ }^{7}$. Accanto alla Casa editrice EDIT, fondata già nel 1952, di cui si dirà nel prosieguo di questo articolo, nel 1963 nasce il Circolo dei poeti, dei letterati e degli artisti, nel 1964 si fonda «La Battana», rivista trimestrale di cultura, che da allora esce con regolarità e sulle cui pagine i poeti, i narratori, i saggisti, i critici letterari istro-quarnerini pubblicano i loro lavori, nel 1968 viene concepito il Centro di Ricerche Storiche di Rovigno, che dà il via alle attività nel $1970^{8}$.

4 Damiani, A., "A contare infine resta la poesia”, in La cultura degli Italiani dell'Istria e di Fiume, ETNIA, Centro di Ricerche Storiche di Rovigno, Università Popolare di Trieste/Unione Italiana di Fiume, Rovigno/Trieste, 1997, p. 217.

5 Damiani, A., "Quel lembo d'Italia oltre confine", in La cultura degli Italiani dell'Istria e di Fiume, op. cit., p. 30 .

6 Milani, N., e Dobran, R., (a cura di), Le parole rimaste. Storia della letteratura italiana dell'Istria e del Quarnero nel secondo Novecento, collana «L'identità dentro», vol. II, Pietas Iulia Pola/EDIT Fiume, 2010, p. 16.

7 Ibidem.

8 È un'istituzione della Comunità Nazionale Italiana di Croazia e Slovenia che dalla fondazione opera nel campo della ricerca nell'ambito della storiografia e della sociologia. È stata fondata dall'Unione degli Italiani dell'Istria e di Fiume con lo scopo di avviare un processo di chiarificazioni e precisazioni sulla storia dell'Istria, che è senza dubbio una delle più complesse e delicate. Dalla fondazione a oggi il Centro ha pubblicato circa trecentomila volumi. Il contributo del Centro è stato particolarmente significativo anche perché ha avvicinato il mondo dei ricercatori e degli studiosi (italiani, croati e sloveni), che hanno accettato il modello aperto di confronto storiografico proposto dall'istituzione. Difatti, il Centro è stato sempre una finestra aperta per dialogare con gli "altri", per confrontarsi con le storiografie croata e slovena, ma anche con quella della nazione d'origine. Nel 1995 la biblioteca del Centro è stata nominata dal Consiglio d'Europa "Biblioteca depositaria del Consiglio d'Europa", e da allora in essa vengono depositate tutte le pubblicazioni dell'organismo europeo attinenti le questioni dei diritti umani e delle minoranze. 
Nell'impossibilitàdipresentare unpanoramaesaustivocheillustricompiutamente le molteplici iniziative e le attività promosse da sodalizi, istituzioni e organismi, l'attenzione di seguito sarà incentrata sull'annuale Concorso d'Arte e di Cultura «Istria Nobilissima». La manifestazione principe della Comunità Nazionale Italiana di Croazia e Slovenia ha sempre attribuito la massima importanza alla produzione letteraria, poetica e narrativa, e artistica, in primo luogo pittorica, degli appartenenti alla Comunità. Il Concorso è stato istituito nel 1967, frutto della proficua collaborazione avviata a partire dalla metà degli anni Sessanta tra 1'Università Popolare di Trieste ${ }^{9}$ e l'Unione degli Italiani dell'Istria e di Fiume (dal 1991 Unione Italiana $)^{10}$. L'istituzione del Concorso è stata sollecitata da quest'ultima, che «si rendeva perfettamente conto che la componente italiana, per la sua collocazione geopolitica, per il suo retaggio culturale, per la sua scelta ideologica, per la sua affermazione sul piano sociale e per la sua proiezione nel tempo aveva bisogno di uno strumento che nella sua duttilità e malleabilità organizzativa fosse adeguato a far leva sulle forze culturalmente più preparate, vivificandole con sempre nuove acquisizioni derivanti da altre forme istituzionalizzate, in modo da dare voce qualificata alle opere artistiche capaci di trasmettere a un pubblico più vasto di quello minoritario le esperienze umane, sociali, culturali e politiche della minoranza» ${ }^{11}$. Il Concorso ha il merito d'aver stimolato e aiutato nel tempo gli operatori culturali della Comunità Nazionale Italiana di Croazia e Slovenia a emergere dall'anonimato per esprimere le potenzialità creative nei seguenti campi: letteratura (poesia e narrativa in lingua italiana e nei dialetti locali, l'istrioto e l'istroveneto), saggistica, musica, teatro, giornalismo, cinema, video e televisione, nonché arti visive (pittura, scultura, grafica, design, illustrazione, arti applicate, fotografia). Le finalità del Concorso, i cui ideatori furono Antonio Pellizzer in rappresentanza dell'Unione degli Italiani dell'Istria e di Fiume e Marcello Fraulini in veste di rappresentante dell'Università Popolare di Trieste, sono evidenziate dagli stessi bandi. I due Enti promotori «ritennero di poter stimolare e valorizzare tutte le forme creative d'arte e di cultura» degli appartenenti alla Comunità Nazionale Italiana, che il Concorso metteva nella «condizione di dare il loro contributo di originalità, di ricerca e di lavoro intellettuale alla società in cui vivono» ${ }^{12}$. Il nome del Concorso, «Istria Nobilissima», che è anche il titolo di un fortunato volume

9 Fin dall'inizio della sua attività, dopo la rifondazione del 1947, l'Università Popolare di Trieste definì i suoi settori d'intervento, incentrati sulla promozione della cultura italiana. Quando nell'autunno del 1954 ebbe la qualifica di Ente Morale, l'Università Popolare divenne il braccio operativo del Ministero degli Affari Esteri della Repubblica Italiana, preposto alla conservazione e alla promozione della cultura degli Italiani dell'Istria, di Fiume e della Dalmazia. Dopo una prima fase in cui si preoccupò di mettere la Comunità Nazionale Italiana in contatto con la cultura della nazione d'origine, l'Ente triestino si attivò nell'opera di tutela e promozione della cultura e della creatività degli italiani d'oltre confine. Questo impegno fu concretizzato nel 1967, quando fu istituito il Concorso d'Arte e di Cultura «Istria Nobilissima».

10 L'Unione Italiana è l'organizzazione unitaria, autonoma, democratica e pluralistica degli italiani delle Repubbliche di Croazia e Slovenia, di cui esprime l'articolazione complessiva dei bisogni politici, economici, culturali e sociali. Finalità principali dell'Unione Italiana sono la salvaguardia e lo sviluppo dell'identità nazionale, culturale e linguistica degli appartenenti alla Comunità Nazionale Italiana (di seguito: CNI), l'affermazione dei diritti specifici, il mantenimento dell'integrità e dell'indivisibilità, l'affermazione della soggettività nonché il conseguimento dell'uniformità di trattamento giuridico e costituzionale della CNI al più alto livello. L'Unione Italiana è legalmente registrata, quale associazione di cittadini, nella Repubblica di Croazia (con sede a Fiume) e nella Repubblica di Slovenia (con sede a Capodistria). Tratto da: http://www.unione-italiana.hr/chi_siamo.htm.

11 Milani, N., e Dobran, R., (a cura di), Le parole rimaste. Storia della letteratura italiana dell' 'stria e del Quarnero nel secondo Novecento, op. cit., vol. II, p. 50.

12 Fraulini, M., Prefazione all'Antologia delle opere premiate al primo Concorso d'Arte e di Cultura «Istria Nobilissima», Unione degli Italiani dell'Istria e di Fiume/Università Popolare di Trieste, Trieste, 1968, p. 6. 
dello scrittore e giornalista triestino Giuseppe Caprin ${ }^{13}$, è stato suggerito da un reperto archeologico, una stele romana collocata all'ingresso del liceo di Rovigno d'Istria.

Dalla sua fondazione a oggi il Concorso, arrivato alla sua quarantacinquesima edizione, è cresciuto e si è modificato in base alle esigenze dettate dal cambiamento dei tempi ${ }^{14}$. È rimasta però intatta la sua funzione primaria: stimolare la partecipazione alla manifestazione di autori e artisti dotati di qualità indiscutibili che attestino la validità di un discorso culturale, e favorire il passaggio graduale dell'eredità etnica agli esponenti delle nuove generazioni, una finalità attuata con l'inserimento nel 1977 della sezione dedicata ai giovani di età compresa tra i quindici e i diciannove anni. Il Concorso ha così realizzato le attese dei fondatori: ha compiuto un meritorio lavoro di raccolta e divulgazione della creatività artistica degli appartenenti alla Comunità Nazionale Italiana, «ha stimolato energie nuove, ha dischiuso più chiare prospettive, ha dato una prima misura sull'intensità e il tono di una presenza che, oltre a fare cultura vuole essere essa stessa cultura» ${ }^{15}$. Senza il Concorso, si può affermare senz'ombra di dubbio, «gran parte dell'attività intellettuale [degli appartenenti alla Comunità Nazionale Italiana] avrebbe stentato a trovare sbocchi adeguati o addirittura sarebbe rimasta tarpata nelle sue aspirazioni senza traguardi» ${ }^{16}$. Una strada lunga e articolata, quella percorsa dal Concorso dal 1967 a oggi, segnata da un iter ciclico scandito dalla pubblicazione dei bandi, dalle cerimonie di premiazione, organizzate di anno in anno in località sempre diverse dell'Istro-quarnerino e, cosa non meno importante, dalla pubblicazione in specifiche antologie di una selezione delle opere premiate, al fine di evitare l'inevitabile dispersione di una creatività che doveva, invece, permanere. Il volume antologico, pubblicato annualmente, offre una sintesi delle opere premiate al Concorso nell'anno precedente. La prima antologia è stata pubblicata nel 1968, e riporta una selezione delle opere presentate alla prima edizione da trentun autori. A oggi sono stati pubblicati quarantadue volumi antologici, che sono un documento ineludibile da consultare per tracciare la cronaca del Premio e la storia di un’operosità intellettuale. Le antologie

\footnotetext{
13 Giuseppe Caprin (1843-1904) è stato giornalista, editore e scrittore triestino. Tra le sue opere ricordiamo: Marine istriane e Lagune di Grado, entrambe del 1890, Tempi andati (1891), Alpi Giulie (1895) e Il Trecento a Trieste (1897). La sua opera più nota, Istria Nobilissima, è uscita postuma. Con uno stile e una lingua piani e con il supporto di un notevole apparato iconografico, l'autore è riuscito a rendere accessibile il testo, ricco di informazioni storiche e geografiche, a una larga fascia di lettori. Al momento della sua pubblicazione l'opera ha rappresentato la più ampia analisi e descrizione dei monumenti storici e artistici dell'Istria.

14 Un'importante novità apportata al Concorso risale al 1992 allorché, in base ad un'iniziativa articolata dall'allora responsabile del Settore culturale dell'Unione Italiana, Silvio Forza, si istituisce il Premio Promozione, che promuove la pubblicazione a parte (al di fuori delle Antologie delle opere premiate al Concorso) di lavori premiati nelle varie sezioni e stimati particolarmente validi. Secondo le disposizioni, il Premio Promozione per la Letteratura, per esempio, può venir assegnato solo ad autori che hanno presentato una raccolta di almeno trecento versi o cinquanta cartelle di prosa. L'opera giudicata migliore fra le sezioni della categoria Arti visive dà invece diritto all'autore a un catalogo personale e a una mostra itinerante in almeno cinque località del territorio istro-quarnerino, nonché ad una mostra a Trieste o in altra località italiana. Nel 1996 si sente il bisogno di ampliare gli orizzonti e di aprire il Concorso anche a chi non è appartenente alla Comunità Nazionale Italiana di Croazia e Slovenia. S'istituiscono, pertanto, due nuove categorie nella sezione Letteratura (narrativa e poesia), rivolte agli esuli e/o ai loro discendenti, nonché ai croati e agli sloveni in possesso di un'ottima conoscenza della lingua italiana. Ai cittadini italiani (esuli e/o loro discendenti) nel bando di concorso si richiede di partecipare con temi pertinenti «il nostro mondo comune (istro-quarnerino e dalmata) nella sua accezione culturale, umana e storica più ampia».

15 Damiani, A., "Valorizzare e divulgare la nostra cultura", in «Panorama», rivista quindicinale, n. 11, EDIT, Fiume, 1982 , p. 5.

16 Ibidem.
} 
testimoniano difatti l'attività creativa e di ricerca di una comunità vitale in modo più che proporzionale alla propria entità numerica. Sono una specola, una sorta di osservatorio, un'immagine ufficiale e istituzionale della sua attività culturale. Nelle pagine delle antologie, che rimangono come traccia preziosa nel tempo e si propongono come lettura della storia culturale della Comunità Nazionale Italiana di Croazia e Slovenia, è custodita la sintesi di un patrimonio di opere che recano i contrassegni inequivocabili e sempre suggestivi della loro originale fisionomia e il respiro inconfondibile della loro terra. Ogni volume presenta aggiornamenti significativi della vita culturale della Comunità, attestandola come vita non marginale. Sono proprio i volumi antologici a conferire al Concorso, specchio della vitalità fattiva di una comunità che mantiene forti legami con la tradizione senza mancare al contempo di percorrere strade di ricerca nuove, «un'impronta del tutto particolare e fanno di esso qualcosa di più e di meglio di una contingente o effimera manifestazione culturale» ${ }^{17}$. Il conferimento dei premi ai vincitori difatti non è fine a se stesso. Con la pubblicazione delle antologie, il Concorso si traduce in «una concreta presentazione di autori e in un'aperta e diffusa circolazione di scritti: in altre parole, un premio vivo, un premio che resta» ${ }^{18}$.

Sfogliando gli indici delle antologie stupisce la cospicuità degli autori che sono stati premiati o segnalati al Concorso, le cui opere sono state inserite di volta in volta nei volumi. Ė un'umanità poliedrica che «ricorda, pensa e spera, muovendosi per lo più nei meandri del privato e nei "termini”" sensuali e territoriali istro-quarnerini» ${ }^{19}$. Il Concorso, senza averlo forse predisposto, ha fatto da intermediario tra «il bisogno latente degli italiani di "cogliere e fermare la percezione di sé", facendo uso della discriminante culturale [...], e la gratificazione di questo bisogno» ${ }^{20}$. È risaputo che una comunità minoritaria che non ha modo di conservare la propria memoria storica, di tramandarla, di esistere e affermarsi in riconoscibili parametri culturali, è destinata prima o poi a soccombere all'assimilazione. In questo senso il Concorso, per quanto possibile, ha corrisposto alle attese: ha fatto della cultura un fenomeno non solo individuale ma sociale, collettivo, storico. Sul piano civile la manifestazione ha contrastato il passo alla strisciante assimilazione degli italiani nell'area istro-quarnerina, sviluppando in essi il legame al territorio d'origine e alla sua storia, che restano centrali, perché da qui essi traggono e comprovano l'identificazione etnica di segno autoctono. Incentivando e promuovendo la creatività artistica, «Istria Nobilissima» ha contribuito a «mantenere viva e rinnovare la cultura e la memoria storica degli italiani dell'Istria e del Quarnero in modo che non si perda mai di vista la fisionomia della cultura nazionale, anche se questa non va pensata in funzione di chiusura ma di apertura alla multiculturalità» ${ }^{21}$.

È impossibile produrre l'elenco degli artisti che dall'anno di fondazione a oggi hanno partecipato al Concorso, il numero supera le millecinquecento unità. Di seguito

17 Maier, B., Prefazione all'Antologia delle opere premiate al settimo Concorso d'Arte e di Cultura «Istria Nobilissima», Unione degli Italiani dell’Istria e di Fiume/Università Popolare di Trieste, Trieste, 1974, p. 6.

18 Ibidem.

19 Milani, N., e Dobran, R., (a cura di), Le parole rimaste, Storia della letteratura italiana dell'Istria e del Quarnero nel secondo Novecento, op. cit., vol. II, p. 55.

20 Ivi, p. 56.

21 Milani Kruljac, N., Prefazione all'Antologia delle opere premiate al quarantaduesimo Concorso d'Arte e di Cultura «Istria Nobilissima», Unione Italiana di Fiume/Università Popolare di Trieste, Trieste, 2009, p. 5. 
nominiamo solo alcuni, in ordine alfabetico. Sono autori e artisti che riteniamo tra i più significativi nel campo letterario e delle arti visive, gli ambiti che in questo discorso c'interessano più da vicino. Nel campo delle lettere (poesia e narrativa in lingua italiana o nei dialetti locali) ricordiamo i seguenti autori: Vlada Acquavita, Marco Apollonio, Libero Benussi, Vlado Benussi, Aljoša Curavić, Gianna Dallemulle Ausenak, Alessandro Damiani, Roberto Dobran, Anita Forlani, Koraljka Leković, Laura Marchig, Nelida Milani, Ester Sardoz Barlessi, Mario Schiavato, Giacomo Scotti, Carla Rotta, Maurizio Tremul, Claudio Ugussi, Ugo Vesselizza, Eligio Zanini. Autore di versi in istrioto, l'antico dialetto della nativa Rovigno d'Istria, con la silloge Mar quito e alanbastro (Mare quieto e limpido) Eligio Zanini è stato il vincitore nel 1967 della prima edizione di «Istria Nobilissima».

Tra i vincitori della sezione Arti visive, le cui opere sono riprodotte annualmente nelle pagine dell'antologia (l'opera che si aggiudica il primo premio o che viene segnalata per meriti particolari è riportata in copertina), vanno ricordati il pittore Mauro Stipanov di Fiume e il maestro della fotografia Virgilio Giuricin di Rovigno d'Istria per aver meritato il maggior numero di riconoscimenti. E ancora, Romolo Venucci ${ }^{22}$, considerato dagli esperti il più importante pittore fiumano del Novecento, maestro dei più affermati pittori istro-quarnerini, al quale Sergio Molesi e Erna Toncinich, consulenti artistici dell'Università Popolare di Trieste e dell'Unione Italiana di Fiume, hanno di recente dedicato un'importante monografia, che va ad aggiungersi a quella già pubblicata nel 1996 da Daina Glavočić. Da nominare inoltre Alfredo Peruško, Umberto Matteoni, Fulvio Juričić e Solidea Guerra di Pola, Gianfranco Miksa, Erna Toncinich, Luciana Hlupar Trinajstić, Loretta Janko e Bruno Paladin di Fiume, Fulvia Zudič di Capodistria e Quintino Bassani di Albona. Il pittore albonese, proiettato nella dimensione nazionale della Croazia e partecipe dell'avanguardia internazionale, è rimasto tuttavia profondamente legato all'ambiente istriano sì da divenire il più autorevole cantore del paesaggio della sua terra, inteso come topografia simbolica della memoria. Un'attenzione a parte merita Claudio Ugussi, poeta, narratore e pittore di Pola, per essere stato nel 1967 il primo vincitore di «Istria Nobilissima» con un'opera di pittura, il dipinto a olio su tela La raccolta del fieno, una «felice trasposizione pittorica che coglie la particolare poesia della terra istriana per mezzo di una sensibilità cromatica equilibrata» ${ }^{23}$. La riproduzione del quadro di Ugussi, posta in copertina, fregia il volume della prima antologia delle opere premiate al Concorso edita nel 1968.

Chi sono gli artisti della Comunità Nazionale Italiana di Croazia e Slovenia sopra citati? Come ha rilevato in uno dei suoi numerosi interventi Sergio Molesi, per anni commissario di giuria a «Istria Nobilissima» per la categoria delle Arti visive, sono artisti «formatisi all'arte come autodidatti o diplomatisi come pittori accademici tra Pola, Fiume, Lubiana, Zagabria, Milano, Perugia, Roma e Venezia nel contesto di un

22 Non a caso la categoria Arti visive di «Istria Nobilissima» porta il suo nome (Categoria Arti visive - Premio Romolo Venucci). Figlio eccellente di quell'ambiente cosmopolita di cui la cultura fiumana della prima metà del Novecento era imbevuta, Venucci (1903-1976) è una figura emblematica, con un ruolo fondamentale sia in ambito culturale sia in quello sociale, oltre che nel campo didattico e pedagogico. L'artista fiumano ha sviluppato la sua personale calligrafia artistica muovendo dai postulati del cubismo e del futurismo, ma anche sulla traccia di movimenti nati in Italia tra le due guerre. Notevole la sua inclinazione verso l'esperimento e l'avanguardia.

23 Fraulini, M., Prefazione all'Antologia delle opere premiate al primo Concorso d'Arte e di Cultura «Istria Nobilissima», op. cit., p. 8. 
proficuo confronto fra la cultura artistica del paese di residenza e quello della nazione d'origine e con la capacità di proiettarsi nella dimensione internazionale, ma nel saldo aggancio alle proprie radici $\gg^{24}$.

Sfogliando i primi volumi delle antologie delle opere premiate, in un primo momento si potrebbe pensare che il Concorso fosse prevalentemente letterario e che la presenza artistica rivestisse una posizione marginale e, in qualche modo, ancillare. Negli anni Ottanta dello scorso secolo la categoria delle Arti visive ha acquistato una maggiore importanza e autonomia all'interno del Concorso, e da allora le riproduzioni delle opere premiate, riportate in copertina e tra le pagine dell'antologia, non hanno svolto più solo una funzione decorativa. Adeguatamente presentate da un testo introduttivo, steso per molti anni con acribia da Sergio Molesi, giudicate da commissioni di esperti, esse hanno ottenuto la giusta attenzione e una necessaria autonomia, divenendo un capitolo a parte, suscettibile di tradursi in un estratto o, magari, in un volumetto.

Se si considera che «Istria Nobilissima» è un Concorso annuale, allora «dobbiamo felicitarci per il fatto che ogni sua edizione riesca a produrre più di qualche risultato originale e di rilievo» ${ }^{25}$. Tutti i protagonisti, cimentatisi nel tempo alla manifestazione nei vari settori dell'Arte, hanno il merito d'aver contribuito a creare un patrimonio artistico-letterario che approfondisce e intensifica l'interazione intellettuale e umana tra il mondo italiano e quello croato e sloveno. Essi hanno creato con tenacia e forza di volontà una cultura che s'inserisce dignitosamente e senza complessi d'inferiorità in un panorama propriamente europeo, accogliente e non escludente, che accetta e rispetta l'alterità, la varietà e la molteplicità.

\section{La Casa editrice EDIT di Fiume e le sue collane}

In un dopoguerra travagliato, quando in conseguenza all'esodo dall'Istroquarnerino di gran parte della popolazione di lingua e cultura italiana anche lo sviluppo della pubblicistica subì una battuta d'arresto, specialmente per la mancanza di intellettuali in grado di dare un seguito alla locale prestigiosa tradizione giornalistica e pubblicistica in lingua italiana, la fondazione della Casa editrice EDIT di Fiume è stata fondamentale per la rinascita e lo sviluppo della stampa italiana nell'aera istro-quarnerina. La Casa editrice è stata fondata nella primavera del 1952 dall'Unione degli Italiani dell'Istria e di Fiume con lo scopo di trattenere nel "luogo d'origine" l'eredità della ricca tradizione pubblicistica e giornalistica italiana ottocentesca e primonovecentesca, e di darle un seguito nelle pur mutate condizioni storiche, politiche e sociali. Attualmente l'EDIT

24 Molesi, S., L'UPT celebra i suoi cent'anni, in «La Battana», trimestrale di cultura, anno XLIII, n. 183, EDIT, Fiume, 2012, p. 37.

25 Guagnini, E., Prefazione all' Antologia delle opere premiate al trentanovesimo Concorso d'Arte e di Cultura «Istria Nobilissima», Unione Italiana di Fiume/Università Popolare di Trieste, Trieste, 2006, p. 8. 
pubblica il quotidiano «La Voce del Popolo $\rangle^{26}$, il quindicinale «Panorama» ${ }^{27}$, la rivista di cultura «La Battana» ${ }^{28}$ e il mensile per ragazzi «Arcobaleno» ${ }^{29}$. Ma la sua attività non si esaurisce qui. La Casa editrice è difatti preposta a ottemperare a tutte le necessità degli appartenenti alla Comunità Nazionale Italiana di Croazia e Slovenia in ambito editoriale. Pertanto si dedica anche alla pubblicazione di libri scolastici e di opere di argomento vario, prettamente letterario.

Dopo un lungo periodo di immobilismo, in cui furono alcune case editrici zagabresi a pubblicare sporadicamente libri in lingua italiana, dall'inizio del nuovo millennio l'EDIT ha dato vita a importanti iniziative editoriali, determinate dall'ansia e dalla volontà di voltare pagina, di chiudere i conti con il secolo breve, di coprire i vuoti lasciati dal passato e di andare alla ricerca del nuovo, per valorizzare al meglio la contemporanea produzione letteraria degli italiani di Croazia e Slovenia. Sulla scia di questa consapevolezza, l'EDIT si è assunta il compito di realizzare scopi precisi e ineludibili, come rileva in un articolo il suo direttore, Silvio Forza: «trovare e recuperare, sistemare e sistematizzare, valorizzare e pubblicare, distribuire e vendere, ma anche promuovere e diffondere $\rangle^{30}$ le opere degli autori istro-quarnerini. A tale scopo, accanto ad altre proposte, l'Ente giornalistico-editoriale ha avviato alcune collane ed ha instaurato una proficua collaborazione con l'editrice triestina Il Ramo d'Oro Editore. Un'iniziativa di particolare rilievo, che favorisce una più ampia diffusione delle opere degli autori istro-quarnerini che in questo modo possono incontrare l'interesse dei lettori in una quarantina di librerie italiane, dove finora, salvo rare eccezioni, i loro lavori non erano mai approdati. Se non attraversa la soglia della pubblicazione, un testo non socializza,

26 Promosso verso la fine dell'estate del 1944, il primo numero del quotidiano esce il 27 ottobre dello stesso anno. Per approfondimenti in merito alla storia e alla politica redazionale del quotidiano si rimanda al II capitolo (Gli anni postbellici 1945-1950) del primo volume dell'opera Le parole rimaste. Storia della letteratura italiana dell'Istria e del Quarnero nel secondo Novecento, già citata in questo intervento, e nello specifico ai sottocapitoli intitolati La cultura di massa in prospettiva socialista, Lo spazio culturale nella «Voce del Popolo» e Forme della comunicazione letteraria nella «Voce del Popolo».

27 Il primo numero appare nelle edicole nel febbraio del 1952. La rivista viene a sostituire, o meglio assorbe, quelle già esistenti: «Donne», «Vie giovanili» e «Tecnica e sport». Il periodico abbraccia disparati settori della vita politica, culturale, sociale, economica, storica, sportiva, prodigandosi al fine di soddisfare le esigenze dei lettori e di approfondire quegli argomenti che nel quotidiano «La Voce del Popolo» vengono spesso solo sfiorati e trattati brevemente e frettolosamente. Importanti sono stati i supplementi letterari, pubblicati dal 1960. I supplementi faranno maturare l'esigenza di dar vita a una vera e propria rivista letteraria, che verrà fondata nel 1964 con il nome di «La Battana».

28 Per ulteriori approfondimenti relativi al lungo percorso della rivista si rimanda al VII capitolo (I nodi del progetto culturale) del secondo volume dell'opera Le parole rimaste. Storia della letteratura italiana dell'Istria e del Quarnero nel secondo Novecento, e in particolare al sottocapitolo intitolato La casa editrice Edit: i libri, "La Battana» e «Panorama».

29 Anche in questo caso, per maggiori informazioni relative al foglio per l'infanzia, si consiglia la consultazione dell'VIII capitolo (Un giornalino per amico) del secondo volume del compendio Le parole rimaste. Storia della letteratura italiana dell'Istria e del Quarnero nel secondo Novecento, e nello specifico il sottocapitolo intitolato Il pluralismo ha i colori dell' «Arcobaleno».

30 Forza, S., "Un bagaglio di esperienze e saperi da applicare e valorizzare all'EDIT", in «La Voce del Popolo», inserto speciale "Salone Internazionale del libro Torino", 31 maggio 2012, EDIT, Fiume, p. 3. La partecipazione al Salone di Torino per Forza è stata l'occasione per una verifica seria, attuata, come spiega nell'esaustivo articolo, dopo «aver allargato la rete di vendita in Croazia, dopo aver individuato un partner triestino (Il Ramo d'Oro Editore), che ci ha concesso di essere finalmente presenti in una quarantina di librerie sparse su tutto il territorio italiano, dopo aver avviato la vendita online dei nostri libri [...], dopo aver realizzato una ventina di book-trailer [...], dopo aver realizzato il primo e-book, cioè Crinale estremo di Nelida Milani, [...] al quale in breve tempo faranno seguito gli altri titoli del nostro catalogo, dopo aver consolidato la nostra presenza nei social network [...], dopo aver raggiunto 23 titoli pubblicati nella collana «Altre lettere italiane» e 5 nella collana «Lo Scampo gigante» (lanciati in oltre un centinaio di presentazioni in Istria, nel Quarnero, a Zagabria e a Trieste)». 
è come se non ci fosse: occorre pertanto che sia trasformato in merce libraria e sia diffuso, affinché il progetto di chi l'ha concepito attinga al proprio scopo, insediandosi nell'animo dell'utenza. Tutti gli sforzi compiuti recentemente dalla Casa editrice e le iniziative portate avanti con successo vanno in questa direzione, sono importanti per rifondare tutte le competenze e le esperienze acquisite nel tempo e adeguarle alle esigenze attuali. È lecito sperare che, grazie a quest'impegno, gli autori istro-quarnerini e le loro opere riescano a trovare il meritato posto anche nel panorama letterario italiano. Le collane inaugurate dall'EDIT a partire dai primi anni del nuovo millennio sono: «Altre lettere italiane», «Lo Scampo gigante» ${ }^{31}$, «La fionda» ${ }^{32}$, «Passaggi $»^{33}$, avviata con Il Ramo d'Oro Editore di Trieste, «L'identità dentro» ${ }^{34}$, «A porte aperte» ${ }^{35}$, «Egzodika $»^{36}, \ll$ Richiami $»^{37} \mathrm{e} \ll \mathrm{Il}$ contapassi» ${ }^{38}$. Di seguito incentreremo l'attenzione su «Altre lettere italiane», la prima collana avviata dall'EDIT nel 2005 con la pubblicazione

31 È una collana che è arrivata al suo quinto volume che, a differenza delle opere edite in «Altre lettere italiane», pubblica i lavori di autori appartenenti alle nuove generazioni, trentenni e quarantenni, che preferiscono confrontarsi con l'oggi e con il mondo, piuttosto che con lo ieri e il (solo) territorio istro-quarnerino. Indichiamo di seguito autori e titoli delle opere: Ginestre sulla costa (2006) di Simone Mocenni; Femminile singolare (2006) di Carla Rotta; A occhi spenti (2008) di Aljoša Curavić; L'altra parte del cielo (2009) di Marco Apollonio; Chiesa di nessuno (2012) di Roberta Dubac. Con questa collana l'EDIT si è proposta lo "sconfinamento" letterario, svincolandosi anche tematicamente dallo stretto mondo istriano e minoritario. "Il progetto della collana prevede che una sorta di ritorno dello "scrittor prodigo" possa suscitare l'interesse di lettori curiosi di comprendere le contaminazioni della nostra lingua e della nostra letteratura con le trame culturali di chi vive vicino a noi e con noi», così si evidenzia il ruolo della collana in Le parole rimaste. Storia della letteratura italiana dell'Istria e del Quarnero nel secondo Novecento, op. cit., vol. II, p. 21.

32 È una collana dedicata alle opere per l'infanzia, ai lettori junior. Finora sono state pubblicate le seguenti opere: $L a$ misteriosa conchiglia di cristallo/Tajanstvena kristalna školjka (pubblicata in edizione bilingue italo/croata, 2005) di Mirella Malusà; Baffolesto e Codacorta (2012) di Carla Rotta e 5 autori $x 16$ storie (2012), una raccolta di sedici racconti di Carla Rotta, Mario Schiavato, Mirella Malusà, Giacomo Scotti e Elvia Nacinovich, autori affermati nel campo della produzione per l'infanzia.

33 La collana, diretta da Francesco Cenetiempo e Silvio Forza, pubblica le opere di autori italiani dell'Alto Adriatico. Nata dalla collaborazione tra Il Ramo D'Oro Editore di Trieste e l'EDIT di Fiume è dedicata a prosatori e poeti appartenenti, con significazioni diverse, alla cultura dell'Istria e del Quarnero. Nella collana sono state finora pubblicate le seguenti opere: Foiba in autunno (2006) di Ezio Mestrovich; Malechiaro (2006) di Gianfranco Sodomaco; Racconti di guerra (2008) di Nelida Milani; Martin Muma (2008) di Eligio Zanini; Fughe e approdi (2010) di Marino Vocci.

34 È una collana dedicata alla saggistica, che si avvale della collaborazione dei ricercatori della Società di Studi e Ricerche Pietas Iulia di Pola. Sono state pubblicate le seguenti opere:, La forza della fragilità. La scrittura femminile nell'area istro-quarnerina: aspetti, sviluppi critici e prospettive (2004), Elis Deghenghi Olujić (a cura di); Le parole rimaste. La storia della letteratura italiana dell'Istria e del Quarnero nel secondo Novecento (2010), Nelida Milani e Robero Dobran (a cura di). Entrambe le opere sono il risultato di un lavoro collettaneo, realizzato con progetti finanziati dal Ministero degli Affari Esteri delle Repubblica Italiana per il tramite dell’Unione Italiana di Fiume.

35 È una collana dedicata ad autori croati e sloveni tradotti in italiano per confermare e rinsaldare i legami storicoculturali tra le due sponde adriatiche. Finora è stata pubblicata l'opera L'Italia agli occhi dei Croati (2005) di Zdravka Krpina.

36 Una vita all'insegna della convivenza: è questa la vita che oggi desiderano i croati e gli italiani che da sempre vivono in stretto contatto in Istria, nel Quarnero e in alcune città della Dalmazia. Non può esserci però autentica convivenza senza reciproca conoscenza. La collana è dedicata a coloro cui il destino ha riservato la rottura definitiva del cordone ombelicale che lega ognuno al proprio luogo natio e a coloro che nutrono dubbi sulle verità assolute, perché lo svolgimento della storia è sempre ricco di sfumature e di variabili. Nel 2008 è stata pubblicata l'opera Zadar. Od bombardiranja do izgnanstva (1943.-1947.) di Giovanni Eleuterio Lovrovich.

37 È una collana degli autori italiani originari dell'Istria, di Fiume e della Dalmazia che vivono in Italia o altrove. Finora è stata pubblicata l'opera Una storia istriana (2008) di Diego Zandel.

38 È una collana inaugurata nel giugno del 2012, dedicata alle opere che raccontano viaggi e paesaggi. La prima opera pubblicata è Itinerari istriani. Sensazioni ed immagini di Romano Farina (1929-2000), per lunghi anni giornalista della Casa editrice EDIT e collaboratore di RadioTelecapodistria, per la quale ha curato bellissimi documentari sulle località istriane, anche quelle meno note, ma pregne di una particolare suggestione. 
del romanzo L'eredità della memoria di Mario Schiavato ${ }^{39}$. Nella collana sono stati finora pubblicati ventitré titoli. L'ultimo in ordine di tempo è il romanzo La città divisa (2011) di Claudio Ugussi. Dell'autore e dell'opera diremo di più nel prosieguo di questo intervento.

«Altre lettere italiane» è nata per pubblicare, divulgare e far conoscere le opere degli autori della Comunità Nazionale Italiana che vive in Croazia e Slovenia. Lo scopo è «diffondere le opere di una letteratura italiana che nella sua alterità, nella sua diversità italo-istriana e italo-fiumana rimane pur sempre letteratura italiana» ${ }^{40}$. Si tratta, come spiega Silvio Forza, «di una collana che vuole promuovere una prosa e una poesia che nascono in un contesto sociale e linguistico non (più) italiano e che proprio per questa ragione sono state e sono espressione di identità, luogo della conservazione e del recupero della memoria, strumento con il quale tramandare un'eredità culturale intima ma allo stesso tempo di gruppo $»^{41}$. Perché produrre letteratura in lingua italiana e nei dialetti locali nell'Istro-quarnerino non significa solo dare sfogo alla propria vena artistica, non è solo una questione individuale. La produzione letteraria degli appartenenti alla Comunità Nazionale Italiana di Croazia e Slovenia ha difatti una valenza sociale e culturale che riguarda l'intera comunità minoritaria. Questa produzione rappresenta un «forte segnale di un'esistenza ancora pulsante, è desiderio di rendere gli "altri" partecipi di un'esperienza tipica da microcosmo», dalla cui particolarità scaturisce però «un'universalità irripetibilmente preziosa»» ${ }^{42}$. Concepire in questo modo il proprio ruolo e la propria creatività artistica, non solo letteraria, per una comunità minoritaria significa assumere un impegno gravoso ma al contempo gratificante, significa assegnarsi il compito «ciclopico, ma irrinunciabile, di provare a intrecciare pazientemente nella "corda" dell'umanità (che risulta tanto più robusta, quante più storie parziali riesce a connettere tra loro) tutte le varie differenze» ${ }^{43}$.

Perché tra le collane avviate dall'EDIT ci interessa proprio "Altre lettere italiane»? Perché per il tramite della collana l'Ente giornalistico-editoriale non sta solo pubblicando e diffondendo le opere letterarie, di poesia e narrativa, degli italiani di Croazia e Slovenia. Con la scelta della veste grafica dei volumi, affidata all'esperienza e alla sensibilità di Liliana Venucci Stefan, responsabile del Settore editoriale, e alla competenza dell'art director Daria Vlahov Horvat, premiata più volte nella sezione design della categoria Arti visive al Concorso d'Arte e di Cultura «Istria Nobilissima», la Casa editrice si è fatta promotrice di un'iniziativa di grande pregio culturale: diffondere i più affermati e premiati artisti (in massima parte pittori) della Comunità Nazionale Italiana di Croazia e Slovenia. I volumi di «Altre lettere italiane» sono difatti prodotti librari che coniugano due ambiti della creatività degli italiani di Croazia e Slovenia, quello

39 È nato a Quinto di Treviso nel 1931, primo di otto figli di una famiglia di contadini coloni la quale, a seguito degli eventi bellici, si trasferì nel 1943 a Dignano d'Istria. In questa cittadina, ricca di tradizioni arcaiche, è cresciuto e si è formato. Pertanto la considera oggi, con l'Istria in genere, sua patria d'adozione. Poeta e narratore, scrittore per l'infanzia e autore di suggestivi resoconti di viaggio, frutto della sua vocazione di alpinista e esploratore, Schiavato ha vinto numerosi e prestigiosi premi letterari, ed è uno dei più fecondi autori istro-quarnerini. L'eredità della memoria, insieme ai romanzi Terra rossa e masiere e Il ritorno, costituisce una vera e propria "trilogia istriana".

40 Così si legge nella presentazione della collana firmata da Silvio Forza, inserita nella bandella.

41 Ibidem

42 Ibidem

43 Bodei, R., La filosofia del Novecento, Donzelli, Roma, 1997, p. 175. 
letterario e quello artistico ovvero, nello specifico, quello pittorico. Con una selezione oculata, che non perde di vista l'argomento dell'opera in questione, per le copertine dei ventitré volumi finora pubblicati nella collana, Daria Vlahov Horvat ha scelto le riproduzioni di altrettante opere pittoriche di artisti istro-quarnerini. Così, il primo volume, il già citato romanzo L'eredità della memoria di Mario Schiavato, presenta in copertina la composizione pittorica Istria 11 di Quintino Bassani ${ }^{44}$, premiata nel 1996 a «Istria Nobilissima». L'ultimo volume, invece, il romanzo La città divisa di Claudio Ugussi, riporta in copertina un dipinto del 2008 dello stesso autore, affermato e stimato pittore oltre che narratore, che raffigura un particolare di Pola, la città che è il soggetto dell'opera, ossia la sua piazza principale, Piazza Foro. Di seguito citiamo alcuni titoli pubblicati nella collana, con l'indicazione dell'autore dell'opera pittorica riportata in copertina: Ester Sardoz Barlessi, Una famiglia istriana (romanzo pubblicato nel 2005, in copertina: particolare di paesaggio istriano, dipinto ad olio di Claudio Ugussi, polese come l'autrice del romanzo); Lidia Delton, Granai de pulvaro/Granelli di polvere (silloge in istrioto pubblicata nel 2005, in copertina: particolare di paesaggio istriano del 1978, di Alfredo Peruško); Gianna Dallemulle Ausenak, Con voce minima (in copertina: Barche al tramonto, particolare del 1974, di Umberto Matteoni, polese come l'autrice della raccolta di racconti pubblicata nel 2005); Mario Schiavato, Il ritorno (romanzo edito nel 2006, in copertina: Istria III, particolare del 2002, di Loretta Janko); Osvaldo Ramous, Lotta con l'ombra e altri racconti (edito nel 2006, in copertina: Da Attila a Lepanto, particolare del 1954, di Romolo Venucci); Vlada Acquavita, Herbarium mysticum. Clausole medievali (silloge del 2007, in copertina: una composizione creata apposta da Erna Toncinich); Nelida Milani, Crinale estremo (raccolta di racconti del 2007, in copertina: Sale AZ 2007, di Fulvia Zudič); Adelia Biasiol, Una voce sommessa (silloge del 2008, in copertina: Tramonto, di Luciana Hlupar Trinajstić); Osvaldo Ramous, Tutte le poesie (raccolta del 2008 di tutte le sillogi del poeta fiumano, in copertina: Figura cubista/Nudo decomposto del 1927, di Romolo Venucci); Osvaldo Ramous, Il cavallo di cartapesta (romanzo edito nel 2008, in copertina un particolare di Fiume: Salita XXX Ottobre, di Romolo Venucci); Ennio Machin, Rimembranze fumane (raccolta di racconti del 2008, in copertina: La Piazza delle Erbe del 1954, di Romolo Venucci); Alessandro Damiani, Ed ebbero la luna (romanzo del 2009, in copertina: Theta ori del 1998, di Mauro Stipanov); Laura Marchig, T(t)erra (silloge del 2009, in copertina: Mio amante del 1997, di Bruno Paladin); Anita Forlani, Scadenze quotidiane (silloge del 2011, in copertina: Faded memories (Memorie sfumate o sfuocate) del 2010, di Solidea Guerra).

Quanto riportato dà la misura dell'importanza culturale del progetto ideato e realizzato dalla Casa editrice con la collana «Altre lettere italiane». Per mezzo di un'oculata politica editoriale, per il tramite delle opere pubblicate nella collana, l'EDIT svolge una duplice funzione: diffonde non solo i testi letterari, di narrativa e poesia, degli autori della Comunità Nazionale Italiana di Croazia e Slovenia, ma fa conoscere pure $\mathrm{i}$

44 Quintino Bassani (1928-2007) è stato il primo artista istriano che, nel secondo dopoguerra, ha conseguito il titolo di pittore accademico all'Accademia di Belle Arti di Zagabria. La sua attività espositiva è stata veramente cospicua. Ha allestito mostre personali in Istria, in Jugoslavia, in Europa ed anche negli Stati Uniti d'America, a New York (1965). È da considerarsi uno dei più autentici cantori del paesaggio istriano, che egli ha saputo interpretare in forme legate alla cultura d'avanguardia, ponendosi tra i più qualificati artisti della Comunità Nazionale Italiana di Croazia e Slovenia. 
nomi e le opere dei più affermati pittori di una comunità che anche sul piano artistico ha dato indubbi esempi di solida portata artistica e di innegabile valore umano ${ }^{45}$.

\section{L'arte di Claudio Ugussi tra pittura e scrittura, tra istrianità e universalità}

Tra gli intellettuali della Comunità Nazionale Italiana di Croazia e Slovenia, Claudio Ugussi è la figura ideale di cui parlare in questa sede innanzi tutto per la sua poliedricità. Pittore, poeta e narratore, premiato a molte edizioni del Concorso d'Arte e di Cultura «Istria Nobilissima» per la narrativa e per la pittura (nel 2003 per la pittura ha ottenuto il Premio Promozione), vincitore nel 2004 del prestigioso premio Scrittura di frontiera di Trieste, Ugussi è uno dei maggiori punti di riferimento per chi voglia conoscere la cultura e l'arte degli italiani di Croazia e Slovenia, esempio eccellente, in verità non l'unico, delle potenzialità creative di molti appartenenti alla Comunità Nazionale Italiana, capaci di sdoppiarsi per esprimersi, con risultati per altro ragguardevoli, in diverse discipline dell'Arte.

Nato a Pola nel 1932, Ugussi ha iniziato la sua attività negli anni Sessanta dello scorso secolo come pittore ${ }^{46}$. Dopo una breve parentesi poetica ${ }^{47}$ è passato alla narrativa, cui è approdato nei primi anni Ottanta attraverso un percorso progressivo, segnato dalla contaminazione e dall'intreccio tra arte figurativa e scrittura quale espressione di massima aderenza al mondo istriano e a ciò che di quel mondo egli ha percepito come specifico e intimamente suo. Dopo aver portato a termine a Zagabria gli studi universitari, nel 1959 si è trasferito a Buie, dove fino al pensionamento ha insegnato materie letterarie nelle scuole medie superiori italiane. Risiede tuttora nella cittadina istriana. Dalle ampie vetrate del suo atelier si gode la vista di un panorama stupendo del paesaggio collinare dell'Istria nord-occidentale, scelta molto spesso quale motivo centrale dei suoi dipinti. Difatti, il paesaggio dell'Alto Buiese, con le sue forme e i suoi colori, ha da sempre impressionato l'artista, che in quell'ambiente ha trovato spunti e

45 Per informazioni più dettagliate relative all'EDIT e alle collane pubblicate dalla Casa editrice fiumana, si rimanda ai siti: www.edit.hr e www.edit.hr/editoria/index.html

46 Il percorso d'arte di Ugussi inizia nel 1954 quando s'iscrive alla Facoltà di Lettere e Filosofia di Zagabria. Nella capitale croata, da esterno, frequenta pure i corsi all'Accademia di Belle Arti. Ma già prima, a Fiume, mentre frequentava il liceo, aveva seguito gli insegnamenti di Romolo Venucci, uno degli artisti fondamentali del ventesimo secolo del capoluogo quarnerino e non solo. Con il supporto finanziario dell'Unione Italiana e della Regione Istriana nel settembre del 2011 alla "Galleria Milotić" di Pola è stata allestita una mostra celebrativa per segnalare i cinquant'anni di attività del pittore polese. Un autentico omaggio a questo autore da parte della sua comunità e di un territorio cui ha dato e continua a dare tanto di sé, della sua creatività, delle sue conoscenze, dei suoi ricordi e sentimenti, e del suo impegno. Nel catalogo che accompagna la mostra, lo storico e critico dell'arte Marino Baldini ha riassunto il percorso di formazione di Ugussi iniziato nei primi anni Sessanta dello scorso secolo. Ripercorrendo i suoi cicli e presentando i suoi lavori più pregnanti, il critico ha confermato il valore dell'operosità e della creatività di un pittore che, come pochi altri artisti istriani, ha tratteggiato il paesaggio e le atmosfere istriane in modo personale, arricchendo di contenuti artistici l'espressione figurativa del suo e del nostro spazio vitale.

47 I componimenti poetici sono stati inizialmente pubblicati nelle raccolte di autori vari, in Poesia 1 e Poesia 2, edite a Fiume rispettivamente nel 1964 e nel 1968 a cura del Circolo dei poeti, dei letterati e degli artisti della Comunità Nazionale Italiana, istituito nel 1963. Nel 1969 a Roma è stata pubblicata la silloge Gli ulivi (Fratelli Palombi Editori), accompagnata da una presentazione di Renzo Frattarolo. Alcune liriche tratte dalla silloge sono state pubblicate nel 1968 sulle pagine della prima Antologia delle opere premiate al Concorso d'Arte e di Cultura «Istria Nobilissima» (pp. 153-156). Citiamo la strofa della prima lirica: Siamo come ulivi / avvinti a questa terra sanguigna / cui giorno per giorno succhiamo / la linfa della vita. 
stimoli per nuove suggestioni, nuove prospettive, nuovi confronti con se stesso. Nei suoi quadri dominano pertanto i colli e i declivi che circondano la cittadina, i campi di ulivi che scendono al mare, $i$ cipressi, i campanili e le chiese di campagna ma anche case vuote e in rovina, villaggi abbandonati e svuotati dagli uomini, così tipici della zona. Nelle sue composizioni pittoriche mancano le figure umane, mentre dominano invece le case diroccate e abbandonate, ampie e voluminose, che simboleggiano il vuoto lasciato dall'esodo. Il paesaggio dell'Alto Buiese, così sublimato, grazie a Ugussi è diventato «parte della storia dell'arte moderna» ${ }^{48}$, come evidenzia il critico d'arte Marino Baldini.

Se l'Alto Buiese e il suo paesaggio sono il soggetto privilegiato delle tele di Ugussi, che si connotano per il loro significato allegorico, non possiamo dire lo stesso per la narrativa. Difatti, già con il primo romanzo, La città divisa, premiato a «Istria Nobilissima» nel 1983, l'attenzione si sposta verso un altro ambiente, quello della nativa Pola, delineato con una prosa realistica e con l'analisi lucida e puntuale della situazione socio-politica che regnava in città nell'immediato secondo dopoguerra ${ }^{49}$. Scritto fra il 1982 e il 1983, «in un periodo in cui la componente italiana istriana si liberava dal giogo del silenzio in cui era stata per decenni relegata sia da parte jugoslava sia da parte italiana» ${ }^{50}$, un silenzio determinato dal divieto di recuperare e trasmettere la memoria dei fatti successivi alla firma del Trattato di Parigi e dall'impedimento di elaborare il tema dell'esodo di molti italiani dall'Istria, da Fiume e dalla Dalmazia, il romanzo è stato pubblicato nel 1991 dall'editore Campanotto di Udine. Nel 2002, dalla Durieux di Zagabria, l'opera è stata pubblicata in traduzione croata (Podijeljeni grad) ${ }^{51}$. Tradotto da Lorena Monica Kmet, in Croazia il romanzo è stato accolto positivamente. Grazie alla traduzione, che svolge sempre un ruolo di mediazione tra genti e culture diverse, «per la prima volta il libro ha messo a conoscenza delle vicende istriane, in primo luogo del dramma dell'esodo degli italiani, un più vasto pubblico della Croazia» ${ }^{52}$. Le vicende di cui tratta l'opera sono difatti in gran parte sconosciute «alla maggioranza croata che vivendo lontano dall'Istria non la conosce, se non come ambita meta turistica» ${ }^{53}$, e sono un mistero anche per le «nuove generazioni istriane che ne hanno sentito parlare ma non hanno potuto obiettivamente informarsi su quanto era realmente accaduto perché il ricordo di esse è tuttora volutamente ignorato o perfino "manomesso" dalla politica e dalla storiografia ${ }^{54}$. Le recensioni dell'opera, apparse sui principali giornali croati,

48 Così Marino Baldini nel catalogo compilato per la mostra allestita a Pola alla "Galleria Milotić" in occasione dei cinquant'anni di attività pittorica.

49 Oltre al romanzo Ugussi ha pubblicato la raccolta di racconti Il nido di pietra (Campanotto Editore, Udine, 1993). Nella raccolta sono confluiti i racconti con i quali ha partecipato a varie edizioni di «Istria Nobilissima», già pubblicati nelle Antologie delle opere premiate (La poltrona, Il pittore, La partenza di Obi, Viaggio di circostanza, Vado a Roma, vieni anche tu), e ancora Mare, vento e bandiere, Incontro all'osteria e Il nido di pietra, che dà il titolo al volume.

50 Eccher, C., Prefazione, La città divisa, collana «Altre lettere italiane», EDIT, Fiume, 2011, p. 9.

51 Va sottolineato che Nenad Popović, editore e redattore della Casa editrice zagabrese, segue da vicino la produzione letteraria della Comunità Nazionale Italiana di Croazia e Slovenia. Con l'EDIT ha pubblicato in edizione bilingue italo/ croata la raccolta di racconti L'ovo slosso/Trulo jaje (1996) di Nelida Milani e la raccolta di racconti Corpi/Tjela (1996) di Marco Apollonio. Nel 2004 lo stesso editore ha pubblicato in traduzione croata Il nido di pietra (Kameno gnijezdo) di Ugussi.

52 Milani, N., e Dobran, R., (a cura di), Le parole rimaste. Storia della letteratura italiana dell'Istria e del Quarnero nel secondo Novecento, op. cit., vol. II, p. 175.

53 Ibidem.

54 Ibidem 
hanno rilevato in modo unanime i suoi meriti e hanno evidenziato che sono pochi $\mathrm{i}$ libri che, come questo di Ugussi, sanno raccontare le grandi tragedie della storia senza fomentare odio e senza scadere nel patetico, avendo innanzi tutto presente la necessità di un sano superamento dei traumi del passato. Un'opera coraggiosa, l'hanno definita i critici croati, che per l'assenza di faziosità e per l'equilibrio con cui l'autore affronta e racconta le vicende storiche stimola alla riflessione su un dramma che molti conoscono in maniera distorta e che altri non conoscono affatto. Le considerazioni dei critici croati ci trovano d'accordo. Difatti, ciò che colpisce è il tono disteso del romanzo e il giudizio equilibrato espresso dall'autore sui fatti storici, che si accorda con l'approccio scelto per trattare una materia quanto mai delicata, svolta senza alcun accento d'acribia, o di rivendicazione, o di rimpianto. Pertanto l'opera può essere considerata a buon diritto «un canto laico e al tempo stesso umano e appassionato dello scontro che diventa incontro tra le diversità $\rangle^{55}$. Nel 2011 l'EDIT di Fiume ha ristampato il romanzo, inserendolo nella collana «Altre lettere italiane». La nuova edizione si avvale della Prefazione di Christian Eccher, ricercatore e lettore di lingua italiana alla Facoltà di Filologia di Novi Sad (Serbia), autore di una tesi di dottorato sulla letteratura degli italiani di Croazia e Slovenia discussa alla Sapienza di Roma ${ }^{56}$. La copertina dell'edizione editiana riporta la riproduzione di un dipinto di Ugussi del 2008, che si presenta nella duplice veste di scrittore e pittore: un particolare di Pola, Piazza Foro, con il tempio di Augusto e il Palazzo del Municipio.

«Siamo la memoria che abbiamo. Senza memoria non sapremmo mai chi siamo» ${ }^{57}$. È una frase di José Saramago. Ci piace citare lo scrittore portoghese per avviare la riflessione incentrata di seguito su La città divisa, il romanzo autobiografico di Ugussi, che si offre al lettore come un mosaico di ricordanze. L'opera è difatti un'ego-storia, la cui fonte è proprio la memoria. Una funzione difficile da maneggiare, la memoria: si perde, può essere lacunosa, ingannevole, incline alla rimozione. Essa non è difatti un archivio di dati ordinatamente raccolti, bensì è un paesaggio vario e complesso, carico di esperienze e di emozioni, dove le tracce di quel che è stato, grandi o piccole che siano, d'indicibile pesantezza o d'inconsistente leggerezza, sono a nostra disposizione. La memoria è pertanto sempre evocazione dell'intimo, di un passato privato riconoscibile attraverso le sue tracce materiali e domestiche, è il racconto frammentato e interiorizzato di temporalità emotive iscritte dentro di noi, che nei ricordi corporei e materiali del tempo cerchiamo rassicurazioni e conferme della nostra incerta e ondivaga esistenza. La memoria è parte di noi e senza non si vive, o si vive male. Essa ha un valore inestimabile, si alimenta della sostanza vitale del passato, spesso indifferente agli scenari algidi della retorica politica e anche della Storia. Sarebbe pertanto quanto mai necessario fare della memoria una forma di educazione permanente, al fine di generare quegli anticorpi indispensabili a combattere il riaffiorare di quelle tensioni che hanno generato le tragedie che hanno accompagnato e segnato in maniera drammatica il cammino dell'umanità.

\footnotetext{
55 Eccher, C., op. cit., p. 15.
}

56 Eccher ha dedicato a Ugussi un corposo saggio intitolato Le molteplici facce della storia: l'Istria di Claudio Ugussi, pubblicato nella rivista «Avanguardia» (n. 43, Roma, 2010), diretta da Francesca Bernardini Napoletano e Aldo Mastropasqua.

57 Saramago, J., in La memoria dell'esilio-l'esilio della memoria, Depetris, G., (a cura di), Edizioni Dell'Orso, Alessandria, 2010. 
Ci sono esperienze che segnano profondamente e verso le quali la scrittura è quasi costretta, come per segreto impulso, ad aprirsi un varco. L'esperienza traumatica della guerra, del dopoguerra e in particolare il dramma delle opzioni e dell'esodo che ha svuotato case e animi, smembrato famiglie e provocato sradicamento e ferite, ha lasciato un segno profondo e indelebile nel ragazzo e di conseguenza nell'uomo che Ugussi è diventato. Come per altri autori istro-quarnerini ${ }^{58}$, anche per lo scrittore e pittore polese l'urgenza di raccontare è stata motivata dal desiderio così umano di dare voce a una memoria a lungo rimossa, dal bisogno di fermare il ricordo per non lasciarlo evaporare, di oggettivarlo con le parole per non vederlo sparire insieme alla vita, dalla necessità di recuperare il tempo per vincere l'oblio di sé e di tutto un mondo che non c'è più, ma che ci appartiene e ci ha forgiati. Narrare la propria memoria, dove si conservano ancora nitide le immagini delle cose, dei luoghi, delle persone e degli eventi, per Ugussi non è solo un modo per recuperarla e curarla, ma è innanzi tutto occasione per raccontare ne La città divisa la propria storia e quella di un'intera comunità, le sensazioni e le ragioni di chi rimase a vivere a Pola dopo l'esodo di gran parte degli abitanti di lingua e cultura italiana. Un esodo che sconvolse per sempre la configurazione etnica della città che, dopo l'inverno del 1947, si trasformò in una città fantasma, spettrale, abitata da una quieta disperazione, perché svuotata dalla gran parte dei suoi cittadini. Una città stravolta nel suo essere e nella sua essenza, segnata da partenze e addii, percorsa da silenzi incredibili che straziarono l'anima di Ugussi, all'epoca adolescente, e che sono rimasti, indelebili, nella sua memoria.

Passando in quei giorni freddi per le vie di Pola non si sentiva che il cupo rimbombare dei colpi del martello, ingigantiti ancor più dalle pareti ormai vuote delle case. Sembrava che tutta la città si preparasse ad allestire un grande funerale e che in ogni famiglia s'inchiodasse una cassa da morto.

Mi rimane un ricordo molto confuso degli ultimi preparativi e di tutte quelle partenze, anche perché c'era realmente una gran confusione e non si sapeva più chi salutare e non si capiva chi partiva e chi restava; ma la partenza dei nonni non la dimenticherò più.

Vista dal molo la nave mi parve immensa. Il colore bruno scuro, quasi nero del suo scafo le conferiva un aspetto tetro e severo appena vivacizzato dalla grande scritta "Toscana" che si poteva leggere sulla poppa in bei caratteri bianchi. Sulla banchina cassoni e mobilia accatastati e gente che andava e veniva. Altri, senza più forza, se ne stavano seduti sulla propria roba, in attesa dell'imbarco.

La grande nave aveva compiuto anche il suo ultimo viaggio. Il vuoto e il silenzio che regnavano nelle case e per le strade sembravano annunziare che i giorni del furore erano passati, che la città ormai s'era rappacificata con se stessa. I pochi passanti che incontrai non fecero che aumentare quel senso di vuoto. L'eco dei loro passi rimbalzava squallido con un suono quasi metallico e finiva col perdersi come inghiottito da un corridoio senza fine ${ }^{59}$.

58 Nel secondo volume de Le parole rimaste. Storia della letteratura italiana dell'Istria e del Quarnero nel secondo Novecento il IX capitolo, intitolato non a caso Le parole della memoria, è dedicato a quegli autori istro-quarnerini che, come Ugussi, hanno espresso nelle loro opere il bisogno di ricordare considerando la memoria quale essenza della natura umana. Dopo la firma del Trattato di Parigi e il conseguente passaggio dell'Istria, di Fiume e della Dalmazia alla Jugoslavia, gli italiani in Istria, come evidenzia Christian Eccher a p.10 della Prefazione a La città divisa, «rimasero soli, schiacciati, per quarant'anni, fra il blocco capitalista e quello comunista, fra l'Europa e l'altra Europa. Lo sforzo per uscire da questa situazione, per ritrovare la voce, è paragonabile solo a quello del barone di Münchausen, che riuscì a salvarsi dalle sabbie mobili afferrandosi e sollevandosi per i capelli. Per recuperare la memoria negata, la CNI [Comunità Nazionale Italiana] scelse il mezzo che più di altri permette di raccontare e di rielaborare il lutto: la letteratura».

59 Ugussi, C., La città divisa, collana «Altre lettere italiane», EDIT, Fiume, 2011, pp. 166, 169 e 171. 
Sono rimaste altrettanto vive nel ricordo dell'autore le discussioni, più o meno accese, che a guerra finita avvenivano tra i membri della famiglia, tra chi intendeva lasciare la città e l'Istria e chi voleva invece restare, come la madre del protagonista del romanzo, che incarna quella di Ugussi, che in una delle solite diatribe con il cognato Mino, convinto sostenitore dell'italianità di Pola e dell'Istria, dichiara perentoriamente: «Io non mi muoverei mai da casa mia, [...] qui sono nata e qui rimango» ${ }^{60}$. Evitando il pericolo di cadere nell'ossessione, l'autore, uno dei "rimasti", cerca di capire e giustificare le posizioni assunte e le scelte compiute dai membri della sua numerosa famiglia, e racconta allo stesso tempo i drammi e i dubbi che hanno travagliato molti polesi in un'epoca in cui chi optava e partiva esule non era bene accolto né ben visto in Italia, e chi rimaneva, invece, in quella che considerava ed era la sua città e la sua terra, veniva guardato con sospetto dal nuovo regime. E se nelle tele di Ugussi mancano le figure umane, come abbiamo evidenziato, non è così per il romanzo, dove incontriamo una vasta galleria di personaggi, uno stuolo di parenti e di interlocutori con la propria storia privata, impegnati nell'incontro/scontro con la grande Storia, che per tutti è drammatica: opprime, annienta, umilia, calpesta. I personaggi, anche quelli secondari, sono tratteggiati con "pennellate" nitide sicché, diversamente che nella pittura, nella narrativa Ugussi mostra d'essere un abile ritrattista.

In un dopoguerra congestionato, quando le sorti della città in mano agli alleati anglo-americani erano ancora incerte e si attendeva la ratifica o l'eventuale (improbabile) revisione del trattato di Parigi, gli scontri, già presenti all'interno delle famiglie, si manifestavano più cruenti «tra i pro-titini e gli anti-titini (coincidenti con le animosità anti-slave e anti-italiane) $)^{61}$, che si affrontavano nelle vie della città proclamando e difendendo le rispettive convinzioni anche con la violenza. Innescando liti e incomunicabilità anche fra chi era stato amico da una vita, tra genitori e figli, tra fratelli e sorelle, questi conflitti minavano i buoni rapporti e la fiducia reciproca tra $\mathrm{i}$ cittadini di Pola, che a guerra conclusa si presenta pertanto "divisa" e smembrata.

Ma è mai possibile, pensavo tra me, che invece di essere contenti che la guerra sia finita tirino fuori delle discussioni che prima mai si erano sognati di fare. Non è che prima non si parlasse di politica in casa, ma $\mathrm{i}$ discorsi assumevano sempre un tono distaccato e ci si lamentava di qualcosa che era al di fuori di noi e delle ingiustizie che bisognava subire senza poter cambiare niente. Ora invece il dialogo diventava polemico anche tra fratello e sorella. Era stata la guerra che aveva cambiato le persone? E adesso che sembrava tutto finito cominciava invece un'altra storia?

Mi chiedevo più volte, ascoltando quelle discussioni, chi avesse ragione e dove stava la verità. Cercando una risposta mi rendevo conto che probabilmente non l'avrei trovata mai e che la verità, intesa come meta da raggiungere, era solo una invenzione degli uomini. Se ciascuno esponendo le sue convinzioni riteneva essere nel giusto e non accettava di prendere in considerazione le idee altrui, che pure potevano, viste da un osservatore imparziale, contenere un fondo di verità, e se ciò accadeva nell'ambito di una famiglia, come potevamo aspettarci che la ricerca, se non altro di un accordo comune, scaturisse dalla più ampia cerchia di una città o di una regione? E saremmo mai stati capaci, travagliati da contraddizioni che ci trascinavamo dentro da secoli, appena usciti da una guerra fratricida che aveva riaperto più profonde piaghe non mai rimarginate, non dico di ricercare assieme un'ipotetica verità, ma almeno di accettare assieme, spontaneamente e con animo sereno la nuova realtà che si andava affermando ${ }^{62}$

60 Ivi, p. 144.

61 Milani, N., e Dobran, R., (a cura di), Le parole rimaste. Storia della letteratura italiana dell'Istria e del Quarnero nel secondo Novecento, op. cit., vol. II, p. 172.

62 Ugussi, C., op. cit., pp. 85 e 136. 
Ugo, l'alter ego dell'autore, ne La città divisa narra le vicende a tratti rocambolesche della propria adolescenza trascorsa negli anni del secondo conflitto fra una Pola colpita da frequenti bombardamenti a tappeto e un paesino situato nel cuore della campagna istriana dove, a causa della recrudescenza della guerra, viene mandato presso alcuni lontani parenti, mentre gli altri due fratelli, più piccoli, sono accolti dai nonni materni e la madre, vedova di un operaio militarizzato scomparso in Grecia, rimane a Pola a lavorare all'Arsenale per sfamare i figli. La permanenza da sfollato del protagonista nel villaggio dell'entroterra istriano si rivela fondamentale. È un'occasione per crescere fisicamente e maturare spiritualmente attraverso un'educazione sentimentale che lo segnerà per tutta la vita. Difatti, nonostante la notevole differenza tra il mondo urbano e l'ambiente marinaresco al quale è abituato (sono molte le pagine dedicate nell'opera al racconto d'imprese per mare, rivelatrici di un'evidente confidenza dell'autore con i libri di Conrad) e quello rurale/slavo che lo ospita, il protagonista, accudito amorevolmente da quella che lui chiama affettuosamente Teta-Tonka e dalle sue figlie, si trova bene in campagna. Cresce e matura, coglie la ricchezza culturale, umana e paesaggistica di un mondo fino a quel momento sconosciuto, che conduce una vita semplice e operosa, sorretta da saldi principi morali e da un profondo sentimento religioso, così diverso da quello che al giovane viene impartito a scuola durante le ore di religione da don Sestan, che «girava come un gatto arrabbiato tra i banchi» ${ }^{63}$, a mollare scapaccioni a chi non sapeva recitare il Credo e non seguiva con attenzione il Vangelo, e scopre finanche le prime pulsioni sessuali.

La fiamma della candela, che avevo posato sul comodino, faceva balenare le loro ombre sulle pareti della camera [...] La visione di quelle braccia candide e rosee e del petto che un'ampia scollatura non tratteneva, le loro cosce piene che mi balenarono un attimo davanti in un movimento incontrollato, continuarono ad accompagnarmi a lungo dopo che Stana aveva soffiato sulla candela [...] Solo adesso mi rendevo conto della differenza con le due donne che vedevo di giorno intente ai lavori anche più duri della terra. [...] E adesso invece la luce tremolante di quella candela mi aveva svelato un altro mondo, che ancora non conoscevo, ma che mi sembrava ancora più fantastico e fascinoso in quanto non immaginabile per me sotto quei vestiti di grezzo tessuto, e ancor più in donne intente tutto il giorno a lavori che comunemente venivano svolti da uomini ${ }^{64}$.

Per le frequenti uscite notturne e per qualche parola colta dai discorsi concitati fatti dinanzi a lui dalle donne sempre a bassa voce, il protagonista intuisce che Tonka e le figlie svolgono attività clandestina, aiutando i partigiani nascosti nei boschi intorno al villaggio, di cui sentiva parlare con insistenza, specialmente la sera intorno al fuoco. Per l'adolescente quei racconti avevano un fascino particolare, accendevano la sua curiosità e alimentavano la sua fantasia. Ascoltando il racconto di quelle gesta leggendarie cresceva in lui l'orgoglio per il fatto che lo zio Alfredo, come molti italiani di Pola e dell'Istria, avesse scelto di combattere al fianco dei partigiani. Così, pensava, adesso «non si poteva più accomunare tutti gli italiani coi fascisti», perché con quella «scelta

\section{Ivi, p. 49.}

64 Ivi, pp. 38 e 39. Nel modo in cui l'autore affronta il tema, ci pare di poter intravedere un'evidente familiarità con la narrativa di Pier Antonio Quarantotti Gambini. In molti suoi romanzi, infatti, Quarantotti Gambini ha descritto con molta delicatezza adolescenti colti, come il protagonista del romanzo ugussiano, nel momento in cui sperimentano l'effetto dei primi impulsi sessuali. Sull'autore capodistriano e il suo opus narrativo Ugussi ha steso una corposa tesi di laurea. 
volontaria molti di essi avevano riscattato almeno in parte il danno arrecato prima da quegli altri» ${ }^{65}$.

Immaginavo i partigiani [...] come esseri vestiti di bianco, quasi dei fantasmi, che correvano di notte sulla neve ad accendere fuochi, una sorta di folletti irraggiungibili che affrontavano senza paura il nemico, per vendicare i contadini soprattutto, e poi si dileguavano nel fitto dei boschi o nel cuore della montagna.

E aumentava così il mio desiderio di vederli quei guerrieri misteriosi, di conoscerli. Erano per la maggior parte istriani, avevo inteso dire, almeno quelli che combattevano in Istria, ma li guidava tutti un capo ormai leggendario, il cui nome correva sempre più frequente sulle bocche della gente, un nome molto breve, Tito, che doveva essere il suo appellativo di cospirazione e di battaglia. Si cantava anche una canzone che parlava di lui e delle sue gesta. Era introvabile, si diceva. I tedeschi avevano fatto di tutto per catturarlo, ma egli sfuggiva sempre agli accerchiamenti per ricomparire altrove e continuare la lotta ${ }^{66}$.

In seguito, quando a guerra finita tornerà in una Pola "divisa" e contesa per riunirsi agli altri membri della sua famiglia, Ugo ripenserà sempre con malinconia alla serenità dei mesi trascorsi presso la famiglia di Teta-Tonka che non avrebbe mai dimenticato, all'ospitalità sincera che gli era stata offerta, a quel mondo slavo genuino che aveva conosciuto e al quale si era affezionato, che nulla aveva di minaccioso, come spesso gli aveva fatto credere chi si nutriva di inutili e sterili pregiudizi e lo considerava inferiore per civiltà e per cultura. Verso quel mondo, conosciuto nell'adolescenza, ossia in un periodo fondante per la formazione di un individuo, egli proverà per sempre una rispettosa simpatia ${ }^{67}$.

E anche quell'antagonismo e quell'insofferenza verso tutto ciò che apparteneva al mondo slavo doveva avere delle motivazioni che non erano sorte per caso in quegli anni. Eppure quel mondo slavo io l'avevo conosciuto almeno in parte, quei mesi che ero stato sfollato, e non avrei mai dimenticato l'ospitalità della famiglia di Teta-Tonka, la loro semplicità e la loro coerenza tra vita di ogni giorno e il loro credo religioso. Essi erano lì da secoli, su quella terra rossa alla quale appartenevano e dalla quale, staccati, non avrebbero potuto sopravvivere; noi eravamo nelle nostre città chissà da quando, parlavamo un'altra lingua, avevamo un'altra cultura e ignoravamo quasi che esistessero o volevamo ignorarlo, guardandoli solo per sorridere alle loro spalle ${ }^{68}$.

Se è fondamentale salvare la propria memoria, è altrettanto importante a un certo punto «salvarsi da quella stessa memoria per non diventarne prigionieri, pena l'impossibilità di vedere la realtà in cui siamo immersi anche da altri punti di vista» ${ }^{69}$.

\section{Ivi, p. 69.}

66 Ivi, pp. 34 e 35. Il brano riporta alla mente atmosfere calviniane, ossia le pagine in cui Pin, l'adolescente protagonista de Il sentiero dei nidi di ragno, immagina la vita dei partigiani, anche per lui eroi senza macchia, che combattevano a difesa dei deboli.

67 Il mondo contadino che Ugussi adolescente conosce e apprezza e che gli fa dimenticare la guerra, la paura degli allarmi notturni, le corse ai rifugi, i bombardamenti continui, è lo stesso mondo terragno che Fulvio Tomizza ha descritto nei suoi romanzi "istriani", in particolare in Materada e ne La miglior vita.

68 Ugussi, C., op. cit., p. 94. A p. 12 della Prefazione a La città divisa Eccher scrive: «Il conflitto tra italiani e slavi, secondo Ugussi, è stato innanzitutto un conflitto di classe, che l'Italia fascista aveva reso ancor più crudele di quello che già era: da secoli gli italiani abitavano le città e i borghi mentre gli slavi occupavano le zone rurali interne dell'Istria. Durante il Ventennio, la diversità culturale e linguistica tra le due etnie che si trovavano nella penisola adriatica era diventata il terreno su cui aveva rapidamente attecchito l'odio razziale tipico dell'ideologia fascista, che in Istria assumeva, ancora una volta, le sembianze di uno scontro etnico».

69 Eccher, C., op. cit., p. 13. Eccher riporta il pensiero espresso a proposito della memoria dallo scrittore Predrag Matvejević, che di Ugussi è amico ed estimatore. 
È quanto è riuscito a fare Ugussi che, al pari di altri autori istro-quarnerini, ha «fatto della letteratura un topos, vale a dire un luogo comune, un punto di incontro con l'altro, in questo caso con l'elemento slavo» ${ }^{70}$. Perché la migliore letteratura «è un dialogo ininterrotto con la diversità, uno sforzo estremo della ragion critica di comprendere le ragioni altrui senza perdere di vista le proprie e senza rinunciare a salvare la propria memoria» ${ }^{71}$. Senza mai trarre conclusioni semplicistiche, il protagonista del romanzo, nonostante la giovane età, individua con lucidità e razionalità le cause che stanno alla base dell'esodo degli italiani da Pola, e rivendica l'oltraggio subito dalla Storia in epoche diverse da tutti gli istriani: italiani, croati e sloveni. L'autore accenna alle contrapposizioni dei due regimi che si sono succeduti in Istria, entrambi totalitari ed egualmente deleteri per il destino dei suoi abitanti. Il regime fascista prima, che nel Ventennio si era impegnato a realizzare la bonifica etnica, decapitando, impoverendo e umiliando le comunità croate e slovene, che nella loro maggioranza erano rimaste salde sul territorio: «In realtà il male peggiore l'aveva portato l'improvvisa espansione del nazionalismo fascista che aveva lasciato subito comprendere quali sarebbero state le nuove prospettive, specialmente per gli abitanti di origine slava» ${ }^{72}$, scrive l'autore. Di seguito, nel secondo dopoguerra, pur avendo proclamato la fratellanza italo-slava, il regime titoista creò le condizioni che spinsero la gran parte della popolazione di lingua e cultura italiana ad abbandonare Pola e l'Istria. Con una visione lucida e disincantata dei fatti e un'analisi degli avvenimenti storici priva di misticismo e sentimentalismo, che prende atto e considera le ragioni degli altri, Ugussi evita il pericolo di rimanere prigioniero della propria memoria e di non sapersene liberare, dando conferma del fatto che le storie di vita vissuta sono le più grandi lezioni di civismo perché trasformano la Storia in coscienza. Alla fine del romanzo, per bocca di Franz, fratello del nonno, che durante il Ventennio fascista si era rifugiato in Dalmazia perché avversava il regime, sentenzia fatalisticamente: «Che cosa volete [...] contro la sorte non si può far niente. La storia cambia, la ruota gira una volta da una parte e una dall'altra» ${ }^{73}$. Come a dire che la grande Storia segue un suo corso, di cui a volte non capiamo il senso. Verso l'Istria e la sua gente, la Storia è stata particolarmente matrigna. Nessuno è stato risparmiato. Secondo come "girava la ruota", in fasi alterne, hanno sofferto tutti: italiani, croati e sloveni. Ce lo ricorda spesso anche Fulvio Tomizza non solo nelle opere di narrativa, ma anche in molti dei suoi illuminanti articoli pubblicati in Alle spalle di Trieste (1995).

Ne La città divisa l'io narrante ricompone i drammi accaduti a Pola (e in Istria) durante la Seconda guerra mondiale e nell'immediato dopoguerra, ed esprime compassione per chi non ha più modo di raccontare: le innocenti vittime di Vergarolla, per esempio, oltre ottanta, in prevalenza donne e bambini, interi nuclei familiari, che il 18 agosto del 1946, in una calda domenica estiva, persero la vita dilaniate dallo scoppio di mine di profondità accatastate su quella che all'epoca, sotto il governo provvisorio degli anglo-americani, era una delle spiagge più frequentate dai polesi. Si cercava di vivere la quotidianità, la "normalità", esorcizzando i ricordi di una guerra appena terminata, scacciando anche in quel modo il pensiero per le sorti di una città contesa,

70 Ivi, p. 14.

71 Ibidem.

72 Ugussi, C., op. cit., p. 142.

73 Ivi, p. 172. 
"divisa" al suo interno, il cui destino era ancora incerto. La spiaggia era affollatissima: vi si stava disputando la Coppa natatoria Scarioni, un evento di grande attrattiva che richiamava solo gente desiderosa di ritagliarsi un momento di svago, dopo le brutture della guerra. La strage, che vestì a lutto la città e sulla quale ancora non è stata fatta piena chiarezza, ha spianato la strada all'esodo degli italiani da Pola. Nel capitolo XVII del romanzo Ugussi ricorda quell'episodio che sconvolse la città, in cui anche la sua famiglia perse un congiunto, Paolo, cugino del protagonista, colpito alla nuca da una scheggia. Particolarmente straziante è il ricordo e la ricostruzione dei funerali delle vittime di quella strage di innocenti, che da molti è stata considerata come un vile attentato intimidatorio, perpetrato nei confronti degli italiani perché lasciassero la città e l'Istria. I funerali, che si mossero dalla cappella dell'ospedale civile, sconvolsero il protagonista, alias Ugussi, tanto che poi per anni non poté più seguire un corteo funebre.

L'esplosione di Vergarolla parve portare la cittadinanza alla realtà del momento, come se quella violenta e improvvisa lacerazione avesse tolto anche l'ultima illusione. Si parlava ancora di ratifica del trattato, di referendum, ma si cominciava anche a parlare di partire; anzi già nel mese di settembre qualcuno cominciò a prepararsi temendo che un'improvvisa decisione di annessione non lasciasse neanche il tempo di abbandonare la città. Ma quanti sarebbero partiti? Chi sarebbe rimasto? Tutto sembrava ancora incerto e una risoluzione definitiva per molti sarebbe stata presa probabilmente all'ultimo momento ${ }^{74}$.

Ma per l'autore azioni altrettanto vigliacche sono state l'eccidio di Sanvincenti, un paese dell'Istria interna, perpetrato nel 1944 a danno dei contadini, in prevalenza slavi, durante il quale i tedeschi uccisero tutti i maschi e bruciarono il villaggio, e le frequenti brutali razzie che le giovani camicie nere compivano nei villaggi per procacciarsi il cibo, privando i contadini del poco che avevano, di cui il protagonista del romanzo è stato più volte impotente e atterrito testimone nel periodo che ha trascorso come sfollato nel paesino istriano. In quelle occasioni quei giovani "guerrieri" ostentavano tutta la loro arroganza e mostravano la loro codardia. Era facile, in fondo, prendersela con donne, vitelli da latte e pennuti indifesi. A conclusione dell'ennesima "battuta di caccia", commosso dai singhiozzi disperati che scuotono il petto di Maria, una delle figlie di Teta-Tonka, e colpito dalla tracotanza di quel drappello di scatenati, il protagonista commenta sarcasticamente: «Hanno compiuto una bellissima missione [...] Bravi questi eroi che sanno "vincere e morire", ma con le galline! ${ }^{75}$. Sensibile agli eventi il ragazzo riflette su quanto accade intorno a lui, interiorizza per ricordare. Con l'arrivo dei partigiani in paese e la grande festa con cui la gente li accoglie, si conclude il soggiorno in campagna di Ugo, che viene riaccompagnato a casa in treno. L'autore ricorda vivamente i momenti essenziali di quel viaggio e descrive in passi densi di viva cromaticità, da pittore qual è, i particolari di un paesaggio che resterà per sempre scolpito nella sua anima.

Il treno intanto filava attraverso la campagna istriana. Dal finestrino, quando il convoglio seguiva qualche lunga curva potevo scorgere la locomotiva e la sbuffata nera di fumo denso che si sviluppava mentre un'acre esalazione mi giungeva alle narici e mi pungeva gli occhi.

74 Ivi, p. 157.

75 Ivi, p. 33. 
Visti dal treno i muretti a secco che dividevano i campi sembravano ancora più numerosi. La terra qui doveva essere ancora più ingrata con l'uomo. I campi coltivati erano di un rosso intenso, disseminati tutti di scaglie bianchissime lavate dalla pioggia. Per lo più erano coltivati a vite. Ogni tanto, in una visione improvvisa, qualche donna vestita di nero intenta a zappare ${ }^{76}$.

La guerra è terminata e la numerosa famiglia di Ugo si ricompone. Per la città e per i parenti di Ugo, per sua madre e i suoi fratelli più piccoli inizia una dura lotta per la sopravvivenza. Senza eccezioni, tutti loro, compreso Ugo, che dopo la morte del padre si sente il responsabile della famiglia, non si perdono d'animo e mettono in pratica l'arte dell'arrangiarsi. L'arrivo in città degli alleati spalanca una parentesi di sollievo per la popolazione ormai stremata dalla povertà e da divisioni interne che la sfibrano. Nell'attesa di decisioni storiche, di conoscere cioè il destino della città, gli scontri fra le parti avverse, come già evidenziato, si fanno sempre più aspri e decisi. Dopo l'esplosione di Vergarolla, di cui sopra, tutto conduce all'epilogo: la maggioranza della popolazione di lingua e cultura italiana in un freddo inverno del 1947 abbandona la città. Anche Ugo, che assiste alla partenza di parenti e amici, considera la possibilità di andarsene e per un momento si fa sordo alle argomentazioni della madre che ha invece deciso di rimanere non solo perché dopo la morte del marito ha attraversato momenti durissimi, e non se la sente di affrontare l'ignoto, ma anche perché ha acquisito una certa coscienza sociale che le permette di comprendere e accettare con fiducia la politica proposta dal nuovo regime. Il romanzo si chiude con pagine commoventi che descrivono lo svuotamento di Pola. Da quel momento in poi la città non potrà più «essere vissuta dai rimasti altrimenti se non come luogo-fantasma, luogo-non luogo perché troppo diverso dall'ambiente domestico nel quale erano nati e avevano vissuto» ${ }^{77}$. I rimasti continueranno a vivere adeguandosi ai cambiamenti imposti dal regime jugoslavo, sempre scissi tra un "prima" e un "dopo", «procedendo nel paragone costante del presente con il trascorso e strutturando la propria identità - come pone in luce il sociologo Maurice Halbwachs - "attorno a riferimenti spazio-temporali che rinsaldano la memoria di un passato comune" $\gg{ }^{78}$.

Per dare voce a giudizi e considerazioni incisive sulla Storia, sulla vita e sui grandi principi dell'esistenza, alla luce del buon senso, lontano da tutte le convenzioni e i conformismi sociali e culturali, in La città divisa Ugussi adotta il punto di vista dell'adolescente che è stato. In questo modo il passato, che secondo Bergson è sempre presente in noi e riaffiora anche quando non ne abbiamo precisa coscienza ${ }^{79}$, è ricostruito nel presente sulla base del ricordo personale della propria adolescenza, e all'interno di quadri di riferimento collettivi. Facendo della memoria un cardine per raccontare il passato e capire il presente, Ugussi insegna che elaborare il passato non è sinonimo di ricostruire. Per quanto ogni elaborazione del passato sia anche, almeno in parte, una sua ricostruzione, l'elaborazione deve innanzi tutto consistere, più che in semplice ripercorrimento del passato, nel suo assorbimento e superamento. Ne La città divisa Ugussi è impegnato nel recupero della memoria personale e collettiva e testimonia allo stesso tempo una fede mai tramontata nell'umano, nell'amore per le persone e

76 Ivi, p. 64.

77 Milani, N., e Dobran, R., (a cura di), Le parole rimaste. Storia della letteratura italiana dell'Istria e del Quarnero nel secondo Novecento, op. cit., vol. II, p. 175.

78 Ibidem. L'opera di Halbwachs cui si fa riferimento è La memoria collettiva, edita nel 1987 da Unicopoli, Milano.

79 L'opera di Henri Bergson cui si fa riferimento è L'evoluzione creatrice, Utet, Torino, 1971. 
per i luoghi non solo dell'adolescenza, ma anche del nostro presente. Così operando, Ugussi assorbe i motivi autobiografici e li trascende in una vicenda che non solo include un'intera città e i suoi abitanti, ma diventa emblematica di un'intera stagione della storia recente. Amicizie, costumi, valori, modalità di interpretare i ruoli familiari, illuminano, per differenza, l'oggi, ponendo sul tappeto una serie di temi che coinvolgono non solo emotivamente, ma anche intellettualmente il lettore, sia che si riconosca nel personaggio/nei personaggi e nelle situazioni per affinità generazionale, sia che voglia addentrarsi in un passato prossimo che è ancora in gran parte sconosciuto, specie alle giovani generazioni. Ugussi è consapevole della necessità di pensare e raccontare non solo per sé, ma per tutti, di ragionare in nome di principi condivisi con altri, di essere un io non singolare ma collettivo, un noi che rende possibile l'intercomunicazione delle esperienze, delle speranze, dei sogni, delle utopie, di fedi comuni sulle quali si fonda la capacità di progetti che devono divenire il collante della vita civile e la spinta verso il futuro. Sorretto da queste convinzioni ne La città divisa l'autore racconta la Storia, quella personale e quella collettiva, in modo piacevole, con una scrittura piana e uno stile capace di alternare, in un tessuto organico, discorsività e forza concettuale, sicché il lettore «non si annoia mai, le avventure di Ugo sono mozzafiato, appassionanti e spesso anche divertenti ${ }^{80}$, mentre i fatti storici «si affacciano in maniera discreta nei dialoghi fra i personaggi e danno a chi legge dei precisi riferimenti cronologici $\rangle^{81}$.

\section{BIBLIOGRAFIA}

BERGSON, H., 1971, L'evoluzione creatrice, UTET, Torino.

BODEI, R., 1997, La filosofia del Novecento, Donzelli, Roma.

DAMIANI, A., 1997, La cultura degli Italiani dell'Istria e di Fiume, collana «ETNIA», Centro di Ricerche Storiche di Rovigno/Università Popolare di Trieste/ Unione Italiana, Rovigno/Trieste.

DEPRETIS, G., (a cura di), 2010, La memoria dell'esilio-l'esilio della memoria, Edizioni Dell'Orso, Alessandria.

MILANI, N., DOBRAN R., 2010, Le parole rimaste, Storia della letteratura italiana dell'Istria e del Quarnero nel secondo Novecento, collana «L'identità dentro», Pietas Iulia Pola/EDIT Fiume.

MONICA L., 2009, La comunità italiana in Istria tra folklore e cultura, in Civiltà italiana e geografie d'Europa, Bianca Maria da Rif (a cura di), EUT Edizioni Università di Trieste.

UGUSSI, R., 2011, La città divisa, collana «Altre lettere italiane», EDIT, Fiume.

80 Eccher, C., op. cit., p. 14.

81 Ibidem. 


\section{KNJIŽEVNOST I LIKOVNA UMJETNOST TALIJANSKE ETNIČKE ZAJEDNICE U HRVATRSKOJ I SLOVENIJI. KNJIŽEVNA I LIKOVNA DJELATNOST CLAUDIJA UGUSSIJA}

U radu se daje prikaz književne i likovne djelatnosti pripadnika talijanske etničke zajednice u Hrvatskoj i Sloveniji. Ta djelatnost i kreativnost posebice dolazi do izražaja u godišnjem natječaju «Istria Nobilissima» koji su 1967. godine utemeljili Talijanska Unija Istre i Rijeke (od 1991. godine Talijanska Unija) i Università Popolare di Trieste (Tršćansko Pučko Učilište). Rezultati toga natječaja zabilježeni su u antologijama koje se godišnje tiskaju od 1968. godine i u kojima su objavljeni odabrani odlomci nagrađenih radova (proznih, pjesničkih, esejističkih i kazališnih), kao i reprodukcije nagrađenih likovnih djela iz sekcije likovne umjetnosti. U radu se ističe i značajna uloga koju ima izdavačka kuća EDIT iz Rijeke, koja je utemeljena 1952. godine i koja ove godine obilježava šezdesetu obljetnicu svojega utemeljenja i rada. Ova je izdavačka kuća od 2005. godine pokrenula niz novih književnih edicija, a u članku se ističe da knjige iz edicije "Altre lettere italiane», (ukupno dvadeset i tri knjige), uz prikaz književnoga stvaralaštva pripadnika talijanske etničke zajednice pridonose i boljem upoznavanju njihovih likovnih djela. To se postiže primjerenim odabirom naslovnica knjiga na kojima su reprodukcije likovnih djela poznatih slikara te zajednice (Quintino Bassani, Romolo Venucci, Bruno Paladin, Alfredo Peruško, Fulvia Zudič, Mauro Stipanov...). U članku autor daje i kritički osvrt na roman La città divisa (Podijeljeni grad) Claudija Ugussija, poznatoga pisca i slikara, rodom iz Pule. Na naslovnici je Ugussijeve knjige, koja je dvadeset $\mathrm{i}$ treće djelo u zbirci «Altre lettere italiane», reprodukcija njegove slike na kojoj je prikazan pulski glavni trg s Augustovim hramom i Gradskom palačom. Ugussi se u knjizi predstavlja u dvojnoj ulozi, kao književnik i likovni umjetnik.

Ključne riječi: književnost, likovna umjetnost, kultura, kreativnost, roman, individualna i kolektivna povijest

\section{LITERARY AND ARTISTIC CREATIVITY OF THE ITALIAN NATIONAL COMUNITY OF CROATIA AND SLOVENIA: CLAUDIO UGUSSI'S EXPERIENCE}

The article deals with the literary and artistic creativity of the Italian National Community (CNI) of Croatia and Slovenia, which expresses itself fully within the framework of the Contest of Art and Culture «Istria Nobilissima», founded by the Università Popolare di Trieste and the Unione degli Italiani dell'Istria e di Fiume (today Unione Italiana) in 1967. The anthologies of the works awarded in the competition, which are issued annually since 1968, are essential to consult in order to learn about the way of emancipation and cultural affirmation that Italians of Croatia and Slovenia have undertaken and followed with determination from the late sixties of last century until today. In the year that the EDIT publishing house in Rijeka, founded in 1952, is celebrating sixty years of its activity, the paper emphasizes the important role that the journalistic-Editorial Board held for the benefit of the Community. It particularly 
highlights the significance of the publication, reprint and dissemination of works of the authors of the Italian National Community of Croatia and Slovenia, especially by means of «Altre lettere italiane», started in 2005 with the publication of the novel L'eredità della memoria by Mario Schiavato. In the essay the author emphasizes the intention to combine the literary and artistic production of Italians, which takes place in the graphical choice of the volumes, realized by Daria Vlahov Horvat. The contribution of the article is also in presenting a critical analysis of the novel La città divisa by Claudio Ugussi, presented to the reader as both narrator and painter. Besides being the author of the work, Ugussi is also the author of the painting that depicts the main square of Pula/ Pola, Piazza Foro, whose reproduction has been placed on the cover.

Key words: literature, painting, culture, creativity, novel, individual and collective history 